\title{
Further engineering of $R$. toruloides for the production of terpenes from lignocellulosic biomass
}

James Kirby ${ }^{1,2}$, Gina M. Geiselman 1,2, Junko Yaegashi, 3,4, Joonhoon Kim 1,4 ${ }^{1,4}$ un Zhuang 1,2, Mary Bao Tran-Gyamfi 1,2, Jan-Philip Prahl1 1,5, Eric R. Sundstrom 1,5 , Yuqian Gao ${ }^{1,10}$, Nathalie Munoz 1,9, Kristin E. Burnum-Johnson 1,9 , Veronica T. Benites ${ }^{1,6}$, Edward E. K. Baidoo $^{1,6}$, Anna Fuhrmann ${ }^{3}$, Katharina Seibel ${ }^{3}$, Bobbie-Jo M. Webb-Robertson ${ }^{1,10}$, Jeremy Zucker ${ }^{1,10}$, Carrie D. Nicora ${ }^{1,10}$, Deepti Tanjore ${ }^{1,5}$, Jon K. Magnuson ${ }^{1,4}$, Jeffrey M. Skerker ${ }^{7,8}$ and John M. Gladden ${ }^{1,2^{*}}$

\begin{abstract}
Background: Mitigation of climate change requires that new routes for the production of fuels and chemicals be as oil-independent as possible. The microbial conversion of lignocellulosic feedstocks into terpene-based biofuels and bioproducts represents one such route. This work builds upon previous demonstrations that the single-celled carotenogenic basidiomycete, Rhodosporidium toruloides, is a promising host for the production of terpenes from lignocellulosic hydrolysates.

Results: This study focuses on the optimization of production of the monoterpene 1,8-cineole and the sesquiterpene a-bisabolene in $R$. toruloides. The a-bisabolene titer attained in $R$. toruloides was found to be proportional to the copy number of the bisabolene synthase (BIS) expression cassette, which in turn influenced the expression level of several native mevalonate pathway genes. The addition of more copies of BIS under a stronger promoter resulted in production of a-bisabolene at $2.2 \mathrm{~g} / \mathrm{L}$ from lignocellulosic hydrolysate in a 2-L fermenter. Production of 1,8-cineole was found to be limited by availability of the precursor geranylgeranyl pyrophosphate (GPP) and expression of an appropriate GPP synthase increased the monoterpene titer fourfold to $143 \mathrm{mg} / \mathrm{L}$ at bench scale. Targeted mevalonate pathway metabolite analysis suggested that 3-hydroxy-3-methyl-glutaryl-coenzyme A reductase (HMGR), mevalonate kinase (MK) and phosphomevalonate kinase (PMK) may be pathway bottlenecks are were therefore selected as targets for overexpression. Expression of HMGR, MK, and PMK orthologs and growth in an optimized lignocellulosic hydrolysate medium increased the 1,8-cineole titer an additional tenfold to $1.4 \mathrm{~g} / \mathrm{L}$. Expression of the same mevalonate pathway genes did not have as large an impact on a-bisabolene production, although the final titer was higher at $2.6 \mathrm{~g} / \mathrm{L}$. Furthermore, mevalonate pathway intermediates accumulated in the mevalonate-engineered strains, suggesting room for further improvement.
\end{abstract}

Conclusions: This work brings $R$. toruloides closer to being able to make industrially relevant quantities of terpene from lignocellulosic biomass.

\footnotetext{
*Correspondence: jmgladden@lbl.gov

${ }^{1}$ Department of Energy, Agile BioFoundry, Emeryville, CA 94608, USA

Full list of author information is available at the end of the article
}

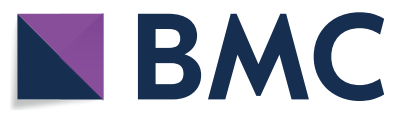

(c) The Author(s) 2021. This article is licensed under a Creative Commons Attribution 4.0 International License, which permits use, sharing, adaptation, distribution and reproduction in any medium or format, as long as you give appropriate credit to the original author(s) and the source, provide a link to the Creative Commons licence, and indicate if changes were made. The images or other third party material in this article are included in the article's Creative Commons licence, unless indicated otherwise in a credit line to the material. If material is not included in the article's Creative Commons licence and your intended use is not permitted by statutory regulation or exceeds the permitted use, you will need to obtain permission directly from the copyright holder. To view a copy of this licence, visit http://creativeco mmons.org/licenses/by/4.0/. The Creative Commons Public Domain Dedication waiver (http://creativecommons.org/publicdomain/ zero/1.0/) applies to the data made available in this article, unless otherwise stated in a credit line to the data. 
Keywords: Rhodotorula, Mevalonate pathway, Isoprenoids, Metabolic engineering, A-bisabolene, Eucalyptol, 1,8-Cineole

\section{Background}

Terpenes, or isoprenoids, constitute one of the largest groups of natural products, playing a variety of diverse roles from steroid hormone biosynthesis in humans to pollinator attraction in plants [1]. Besides the many applications that terpenes play in health and nutrition, they have also attracted a considerable amount of attention as candidates for renewable fuels and bioproducts. Monoterpenes such as pinene and 1,8-cineole show promise as potential precursors to renewable jet fuels [2]. A number of $C_{15}$ sesquiterpenes such as $\alpha$-bisabolene [3] and farnesene [4] can function as diesel fuels, following reduction to their respective alkanes by hydrogenation. Other terpenes such as the $\mathrm{C}_{20}$ diterpene, ent-kaurene may provide bio-based replacements for petrochemicals with a variety of industrial applications [5].

This work builds upon previous efforts to produce 1,8 -cineole and $\alpha$-bisabolene in the single-celled basidiomycete, Rhodosporidium toruloides. This fungus has previously been identified as an attractive host for production of biofuels and bioproducts from lignocellulosic biomass, in part due to its ability to co-metabolize multiple carbon sources, including $\mathrm{C}_{5}$ and $\mathrm{C}_{6}$ sugars and aromatic compounds found in lignocellulosic hydrolysates [6].

1,8-Cineole, or eucalyptol, is the major constituent of eucalyptus oil, but is also produced by several other plants and an endophytic fungus, Hypoxylon $s p$. [7, 8]. Research into the use of eucalyptus oil as a fuel goes back more than three decades, when its suitability for addition to gasoline-ethanol blends was examined. More recently, representatives of the aviation industry announced plans to expand its scope to use in jet fuel, prompting an increased interest in microbial production of 1,8-cineole from lignocellulosic feedstocks [9]. Monoterpene titers in microbial hosts are typically lower than titers achieved for other classes of terpene. Although titers of around $0.5 \mathrm{~g} / \mathrm{L}$ have been reached in Escherichia coli for the monoterpenes limonene [10], 1,8-cineole, and linalool [11], much lower titers are usually observed in fungal hosts such as Saccharomyces cerevisiae [12]. Previous efforts to produce 1,8-cineole from lignocellulosic biomass in $R$. toruloides by expression of HYP3 from Hypoxylon sp. E7406B resulted in a titer of $35 \mathrm{mg} / \mathrm{L}$ [2].

Research on microbial production of terpenes has been more heavily weighted towards sesquiterpenes, which are generally less toxic to microbes than monoterpenes and leave the cell more readily compared to larger terpenes such as carotenoids. Production of $\alpha$-bisabolene was reported in S. cerevisiae and $R$. toruloides at titers of $900 \mathrm{mg} / \mathrm{L}$ and $680 \mathrm{mg} / \mathrm{L}$, respectively [3, 6], with the latter produced from lignocellulosic hydrolysate. Somewhat surprisingly, the diterpene ent-kaurene was produced at higher titers $(1.4 \mathrm{~g} / \mathrm{L})$ from lignocellulosic hydrolysate in $R$. toruloides, perhaps owing to the fact that the diterpene precursor, geranylgeranyl pyrophosphate (GGPP) was enhanced by expression of a heterologous GGPP synthase in the engineered strain [5]. In contrast, production of $\alpha$-bisabolene and 1,8-cineole has thus far relied on native levels of their respective precursors, farnesyl pyrophosphate (FPP) and geranyl pyrophosphate (GPP). GPP in particular is produced in limited amounts in $R$. toruloides as the longer prenyl phosphate precursor FPP is generated in a single step from the mevalonate pathway $\mathrm{C}_{5}$ products, isopentenyl pyrophosphate (IPP) and dimethylallyl pyrophosphate (DMAPP). It therefore seems likely that 1,8-cineole titers in $R$. toruloides are limited by GPP levels and that production of all classes of terpenes may benefit from engineering to increase metabolic flux to IPP and DMAPP. Successive cycles of design, build, test, and learn (DBTL) were applied to explore these limitations to high-level terpene production in $R$. toruloides.

\section{Results and discussion}

\section{Enhancing a-bisabolene titer in $R$. toruloides}

Previous work on engineering $R$. toruloides for production of $\alpha$-bisabolene indicated that flux through the native mevalonate pathway is relatively high in this species. A strain modified only by insertion of a heterologous $\alpha$-bisabolene synthase gene (BIS) from Abies grandis under control of the native $R$. toruloides GAPDH (glyceraldehyde 3-phosphate dehydrogenase) promoter into WT $R$. toruloides achieved $\alpha$-bisabolene titer of $294 \mathrm{mg} / \mathrm{L}$ in a defined medium containing $2 \%(\mathrm{w} / \mathrm{v})$ glucose and $680 \mathrm{mg} / \mathrm{L}$ in a 2-L bioreactor fed with corn stover hydrolysate [6]. Since heterologous DNA was introduced into $R$. toruloides by ATMT, which results in DNA integration at random loci with a variable copy number, it was of interest to investigate the correlation between copy number and $\alpha$-bisabolene titer for a range of $\mathrm{P}_{\text {GAPDH }}$ - $\mathrm{BIS}$ transformants. A good correlation $\left(R^{2}=0.93, p=1 \mathrm{e}-11\right)$ exists between $\alpha$-bisabolene titer and $\mathrm{P}_{\text {GAPDH }}$-BIS copy number for the 20 strains examined and, since a plateau did not appear to have been reached, it seemed likely that BIS expression remained a limiting 
factor for $\alpha$-bisabolene production (Fig. 1a). The linearity of this correlation also suggests that insertion locus may be less important than copy number in determining the level of heterologous gene expression. To test the hypothesis that the $\alpha$-bisabolene titer is still limited by BIS gene expression, the highest-titer $\mathrm{P}_{\text {GAPDH }}$-BIS strain, BIS3, was selected for addition of a second expression cassette consisting of BIS under control of the native $R$. toruloides ANT (adenine nucleotide translocase) promoter [15]. This resulted in strain, GB2, which produced 1.5 -fold more $\alpha$-bisabolene than the parent strain, BIS3, and contained 6 copies of the $\mathrm{P}_{A N T}$-BIS cassette in addition to the original 10 copies of the $\mathrm{P}_{G A P D H^{-}}$BIS cassette in BIS3 (Fig. 1b).

\section{Scale up of a-bisabolene production in lignocellulosic hydrolysate}

$R$. toruloides has been identified as a promising host for production of renewable biofuels and bioproducts because it can efficiently utilize mixed carbon sources and tolerate potential growth inhibitors often found in lignocellulosic hydrolysates [6]. We therefore wanted to determine how the new high BIS-copy GB2 strain performs when grown on a lignocellulosic hydrolysate derived from corn stover (DMR-EH) for $\alpha$-bisabolene production [13]. Growth and productivity were measured in 2-L bioreactors and the impact of nitrogen source and $\mathrm{pH}$ were investigated. Two of the bioreactors (A6 and $\mathrm{A} 7$, respectively) were used to compare two nitrogen sources supplementing the DMR-EH base medium: complex (10 g/L yeast extract) and defined ( $5 \mathrm{~g} / \mathrm{L}$ ammonium sulfate). The medium supplemented with defined nitrogen also contained $100 \mu \mathrm{M}$ iron sulfate and $100 \mathrm{mM}$ potassium phosphate ( $\mathrm{pH}$ 6.0). Medium $\mathrm{pH}$ was maintained above 5.0 during growth in these two bioreactors, while a third culture (A8) was grown in the defined nitrogen DMR-EH medium with no $\mathrm{pH}$ control. Although the $\mathrm{pH}$ in the third bioreactor dropped significantly $(\sim \mathrm{pH}$ 3 ) over the course of the run, the final $\alpha$-bisabolene titer, $1.9 \mathrm{~g} / \mathrm{L}$, was close to that of the $\mathrm{pH}$-controlled cultures (Fig. 2). The two $\mathrm{pH}$-controlled cultures grown on defined nitrogen and complex nitrogen also reached similar $\alpha$-bisabolene titers, 2.1 and $2.2 \mathrm{~g} / \mathrm{L}$, respectively. The $\mathrm{pH}$ in the culture supplemented with yeast extract rose slightly to around $\mathrm{pH} 6.0$ during the first half of the fermentation, only dropping as low as $\mathrm{pH} 5.0$ by the end of the fermentation, while the culture supplemented with ammonium sulfate required an adjustment to keep the $\mathrm{pH}$ above 5.0 from days 2 to 8 (Fig. 2b).

\section{Transcriptomics and proteomics analysis of a-bisabolene producing strains}

Generation of strains that produce different tiers of $\alpha$-bisabolene provides an opportunity to understand how $R$. toruloides respond to the diversion of carbon flux toward a heterologous product and to identify potential targets for metabolic engineering. To examine this response on a systems level, wild-type $R$. toruloides and four strains that produced $\alpha$-bisabolene at various titers (Fig. 1b) were selected for global proteomic and transcriptomic analysis. Growth and sugar consumption were monitored in SD medium containing $10 \mathrm{~g} / \mathrm{L}$ glucose and
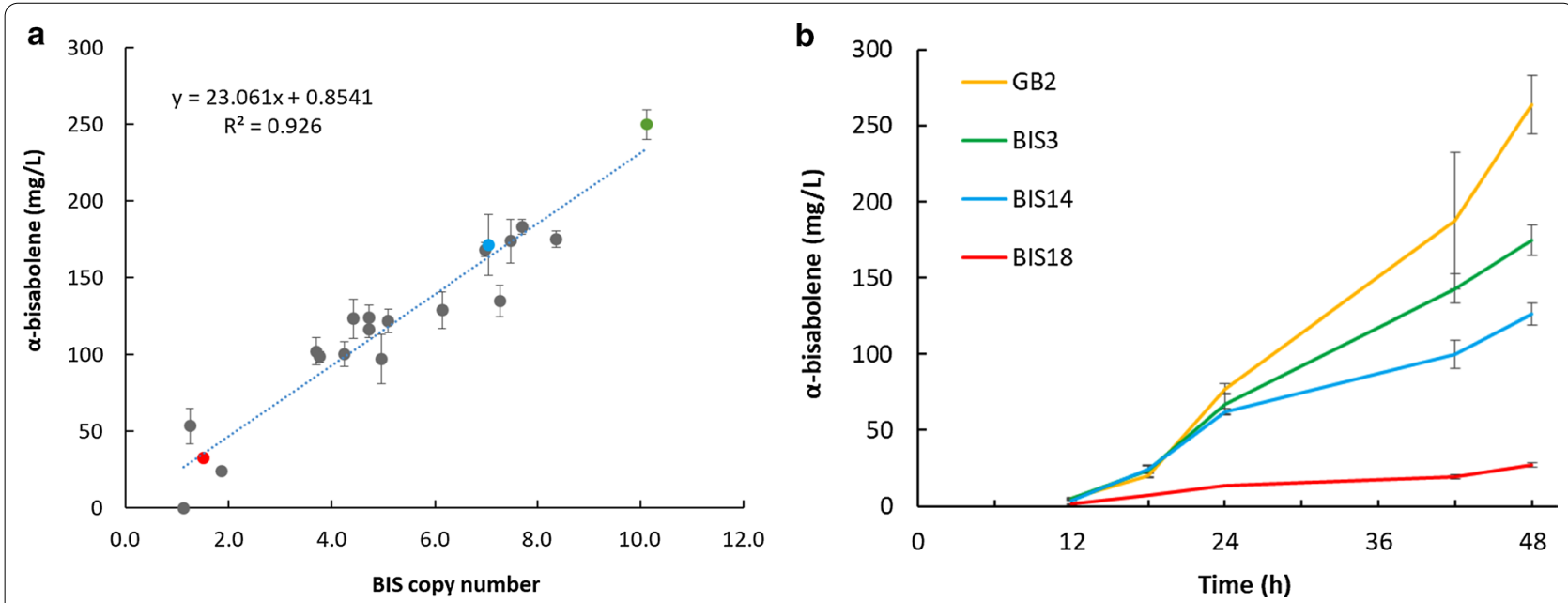

Fig. 1 Engineering the production of a-bisabolene in $R$. toruloides. a Correlation between $P_{G A P D H}$ BIS copy number and a-bisabolene titer at day $8 \mathrm{in} \mathrm{SD}$ medium containing $20 \mathrm{~g} / \mathrm{L}$ glucose. b Production of a-bisabolene in SD medium containing $10 \mathrm{~g} / \mathrm{L}$ glucose by three strains harboring the $P_{G A P D H}-B I S$ cassette at various copy numbers (shown as points of corresponding color in $A$ ) and strain GB2, built by insertion of 6 copies of $P_{A N T}$-BIS into strain BIS3. BIS, a-bisabolene synthase from Abies grandis; $P_{G A P D H}, R$. toruloides glyceraldehyde 3-phosphate dehydrogenase promoter; $P_{A N T} R$. toruloides adenine nucleotide translocase promoter 

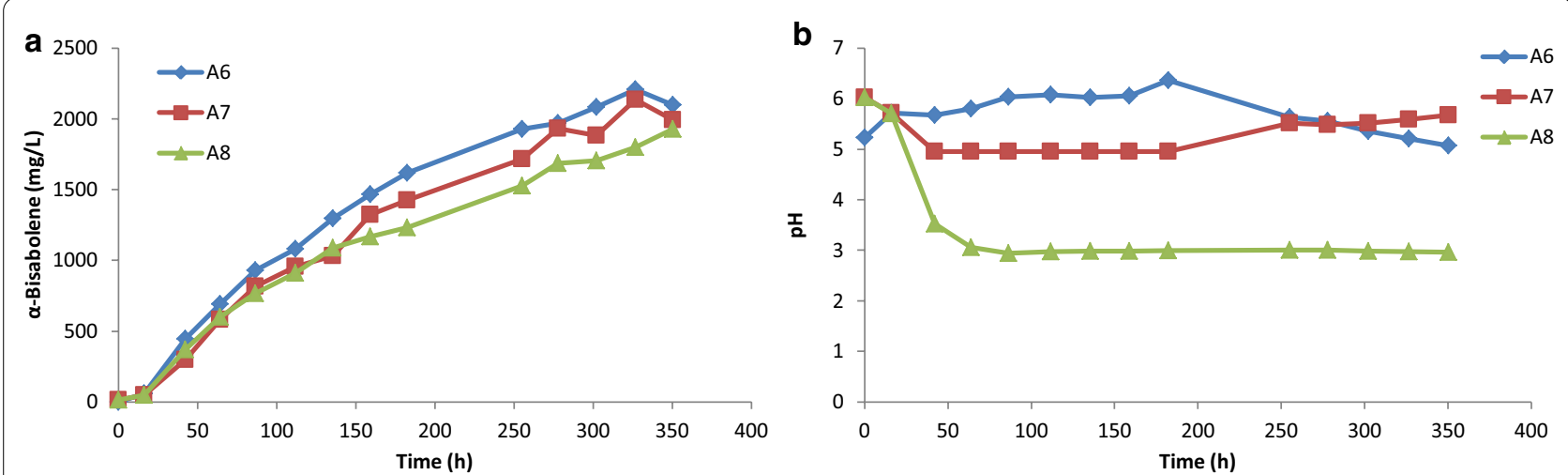

Fig. 2 Production of a-bisabolene at 2-L scale. Strain GB2 was grown in medium containing DMR-EH supplemented with either $10 \mathrm{~g} / \mathrm{L}$ yeast extract (bioreactor A6) or $5 \mathrm{~g} / \mathrm{L}$ ammonium sulfate (bioreactors A7 and A8). Bioreactors A6 and A7 received a sodium hydroxide feed if the $\mathrm{pH}$ dropped below 5.0, while reactor A8 received no pH control. a-bisabolene titer (a) and pH (b) were monitored for 14 days. DMR-EH, Lignocellulosic hydrolysate prepared from corn stover by deacetylation and mechanical refining followed by enzymatic hydrolysis

samples taken at 18 and $48 \mathrm{~h}$ were used for omics analysis (Additional file 1: Fig. S1). Both protein and transcript data indicate that BIS is one of the most highly expressed genes in the highest-titer strain, GB2, harboring both $\mathrm{P}_{\text {GAPDH }}$-BIS and $\mathrm{P}_{A N T}$-BIS (Fig. 3 and Additional file 1: Table S1). Comparing the strains, BIS transcript and protein levels increase in parallel with the increase in copy number and $\alpha$-bisabolene titer, surpassing expression levels of two of the strongest native genes, ANT and TEF1 (translation elongation factor $1 \alpha$ ), in most cases (Additional file 1: Table S1). Interestingly, expression of the first two enzymes of the mevalonate pathway, acetyl-CoA acetyltransferase (ERG10) and 3-hydroxy3-methylglutaryl-CoA (HMG-CoA) synthase (ERG13), also increase in proportion to $\alpha$-bisabolene production, suggesting that the cell induces regulation to increase metabolic flux into the mevalonate pathway to accommodate the diversion of carbon away from native mevalonate pathway products to $\alpha$-bisabolene. The same is true of the native FPP synthase (ERG20), which supplies the precursor to $\alpha$-bisabolene and as well as being a key control point in ergosterol biosynthesis. The expression of other pathway genes underwent more modest changes in expression, even decreasing slightly in GB2, suggesting that they may be good targets for metabolic engineering to enhance flux through the mevalonate pathway. For example, transcript and protein levels for mevalonate kinase (MK) decreased slightly while phosphomevalonate kinase (PMK) changed little in strain GB2 compared to WT (Fig. 3 and Additional file 1: Table S2).

Also notable was the upregulation of ATP citrate lyase (ACLY), acetyl-CoA carboxylase (ACC1), and fatty acid synthase (FAS2) in GB2, relative to WT, possibly responding to changes in acetyl-CoA levels. An upregulation of sterol metabolism genes such as squalene monooxygenase (ERG1), lanosterol synthase (ERG7), and lanosterol 14-alpha-demethylase (ERG11) was also observed (Additional file 1: Table S2).

\section{Enhancing 1,8-cineole production in $R$. toruloides by increasing GPP supply}

In this study, we attempt to optimize production of both $\alpha$-bisabolene and 1,8-cineole. Before attempting to optimize the mevalonate pathway for both terpenes, we first wanted to ensure that there were sufficient pools of the pyrophosphate precursors for these two products. While the $\alpha$-bisabolene precursor FPP is a central metabolite in the ergosterol pathway, 1,8-cineole is made from GPP, which previous work indicates is significantly more limited in R. toruloides [2]. Similar to S. cerevisiae and other fungi, $R$. toruloides lacks a dedicated GPP synthase (GPPS), and FPP is made directly from the $\mathrm{C}_{5}$ precursors, IPP and DMAPP by the enzyme ERG20. Therefore, the initial strategy for engineering higher 1,8-cineole titers focused on optimizing and balancing expression levels of the two terminal enzymes: GPPS and 1,8-cineole synthase. Engineering a high monoterpene titer requires a balance between GPPS and terpene synthase activities that provides sufficient flux to accumulate the target product while avoiding growth-inhibitory levels of GPP $[11,27]$. Promoters for these synthases were selected from native $R$. toruloides genes. Three promoters, ANT, $G A P D H$, and TEF1 were selected based on their relative strength, constitutive expression profiles, and utility in prior work $[2,3,5,15] . \mathrm{P}_{A N T}$ and $\mathrm{P}_{G A P D H}$ were used to express HYP3 from Hypoxylon sp. E7406B, encoding a 1,8-cineole synthase previously identified as a promising enzyme for monoterpene production in $R$. toruloides 


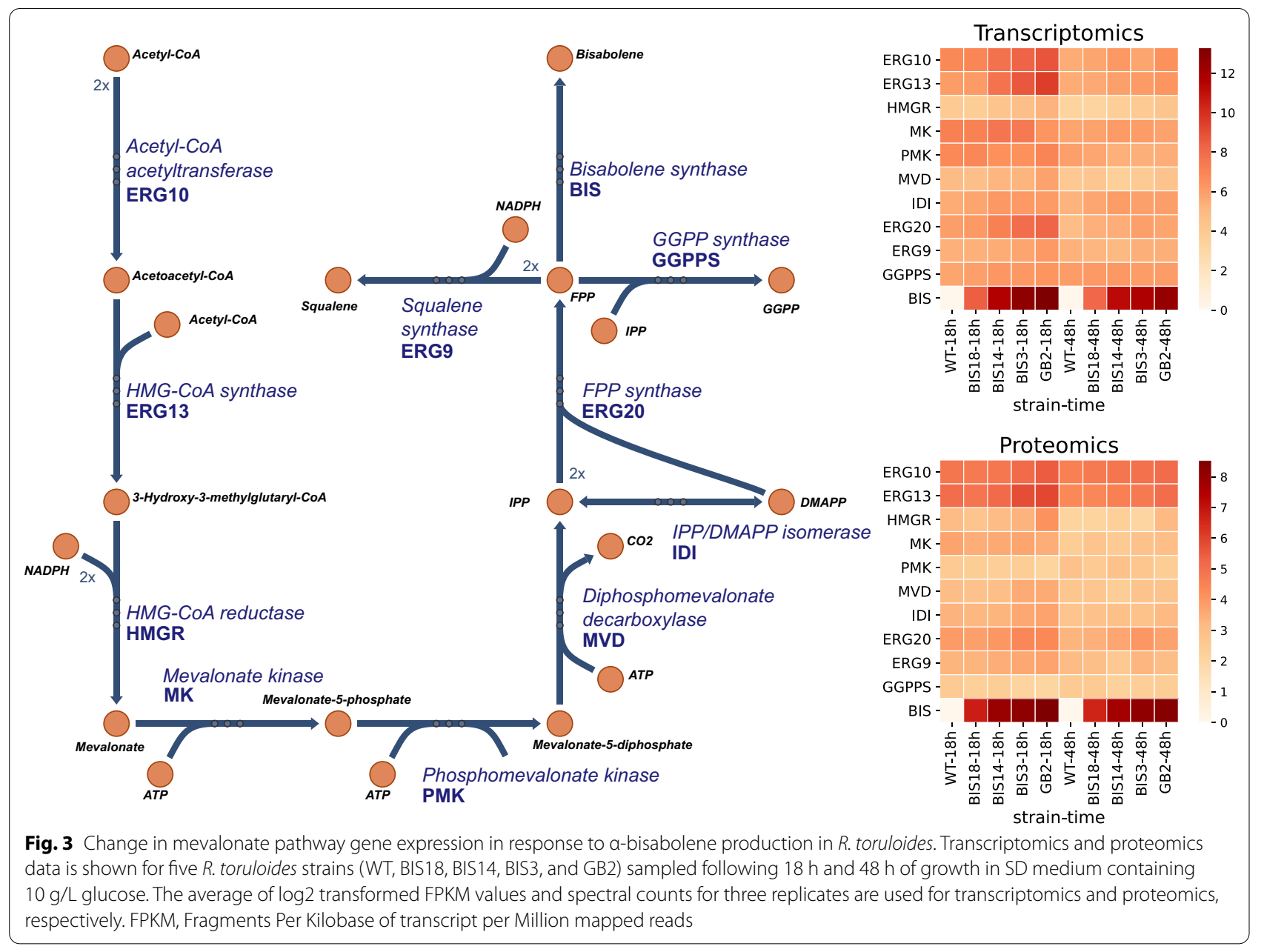

[2]. $\mathrm{P}_{T E F 1}$ was used to drive candidate GPPSs, several of which are FPP synthases containing mutations that alter the prenyl phosphate product chain length specificity in favor of GPP. Genes were transformed into $R$. toruloides by ATMT in either stepwise fashion (gene stacking) or by the use of single, combined constructs. Following each transformation event, up to 40 strains were screened for 1,8-cineole production and the best strains were retested in triplicate in $\mathrm{YPD}_{10}$ medium. The highest-titer strain resulting from each transformation was selected for comparison and further strain engineering.

Various configurations of HYP3 were combined with a GPPS from $A$. grandis with the N-terminal plastid transit peptide removed (tAgGPPS2) or not (AgGPPS2), under control of the TEF1 promoter (Fig. 4a). Each of these strains demonstrated some improvement over the previously published 1,8-cineole titer of $35 \mathrm{mg} / \mathrm{L}$, suggesting that GPP synthesis is limiting to some extent [2]. Combining $\mathrm{P}_{T E F 1}-A g G P P S 2$ with $\mathrm{P}_{A N T}-H Y P 3$ (strain 350) resulted in a 1,8-cineole titer of $58 \mathrm{mg} / \mathrm{L}$ and switching the $H Y P 3$ promoter from $\mathrm{P}_{A N T}$ to $\mathrm{P}_{G A P D H}$ did not change the titer significantly (strain 352, producing $66 \mathrm{mg} / \mathrm{L} \mathrm{1,8-cineole).} \mathrm{A} \mathrm{comparison} \mathrm{of} \mathrm{strains} \mathrm{har-}$ boring both $\mathrm{P}_{G A P D H^{-}}-H Y P 3$ and $\mathrm{P}_{A N T}-H Y P 3$ with either $\mathrm{P}_{T E F 1}-\mathrm{t} A g G P P S 2$ or $\mathrm{P}_{T E F 1}-A g G P P S 2$ indicates that the truncated GPPS (strain 354, $73 \mathrm{mg} / \mathrm{L} 1,8$-cineole) performed slightly better than the full-length version (strain $349,52 \mathrm{mg} / \mathrm{L}$ 1,8-cineole).

Success with engineering monoterpene production has been reported by the use of FPP synthases harboring mutations that alter the substrate-binding pocket such that prenyl phosphate chain length elongation beyond $\mathrm{C}_{10}$ (GPP) is significantly reduced [11, 28-30]. Strains were constructed to compare various mutant FPP synthases, all under control of $\mathrm{P}_{T E F 1}$ and combined with $\mathrm{P}_{A N T}-H Y P 3$, and all but one of them produced significantly more 1,8-cineole than the strain harboring the plant GPPS, $\mathrm{P}_{T E F 1}$-tAgGPPS2 (Fig. 4b). Mutants of the $S$. cerevisiae FPP synthase (ERG20) proved to be more effective than the corresponding mutants of the native $R$. toruloides ERG20, while the most promising enzyme of those tested was GgFPS(N144W) from Gallus 

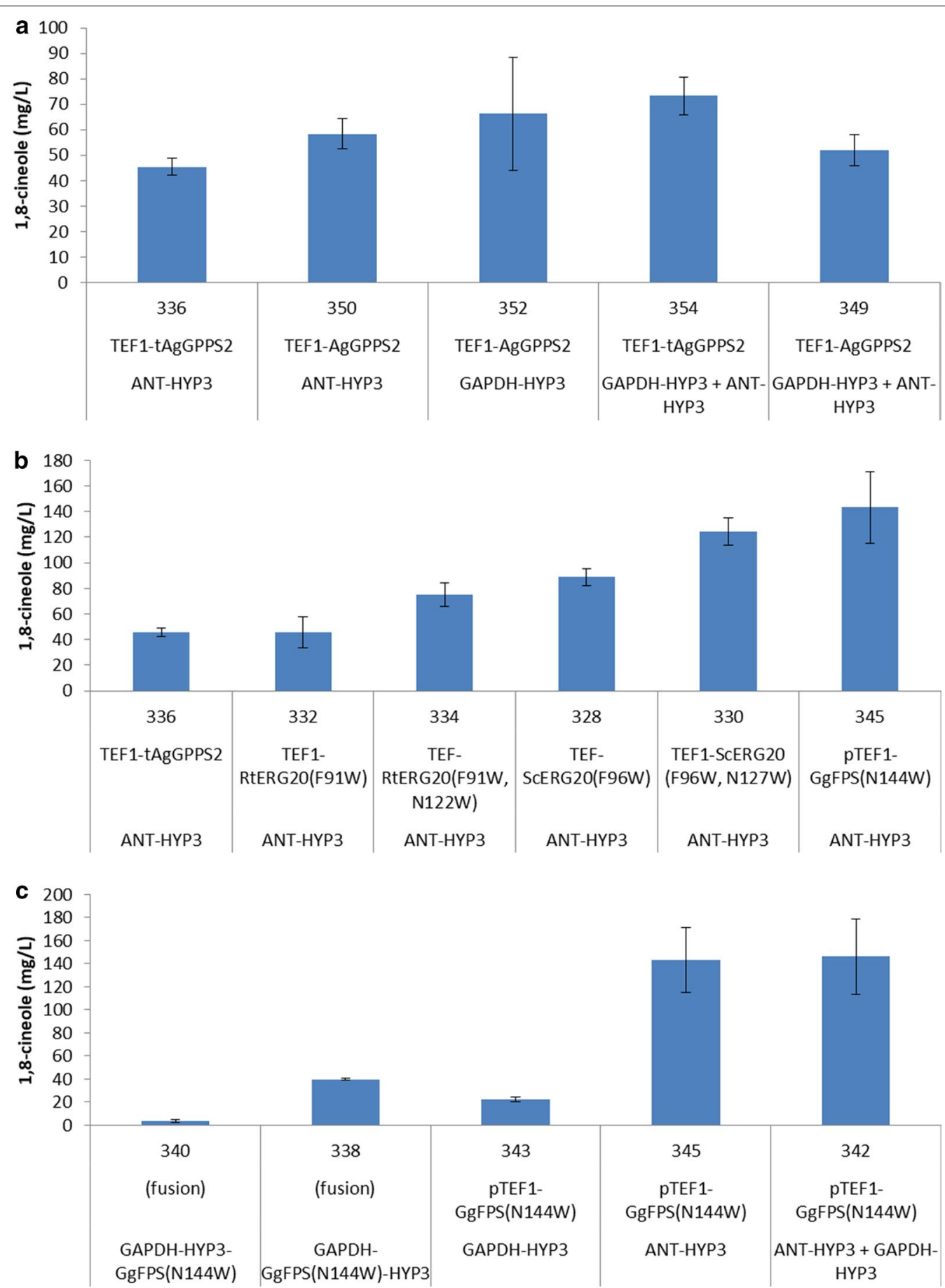

Fig. 4 Engineering 1,8-cineole production in R. toruloides. Strains are described in Table 1. a Various combinations of HYP3 and AgGPPS2. b Different GPP synthases combined with $P_{A N T}-$ HYP3. c Assorted strategies for expression of GgFPS(N144W) and HYP3, individually and as fusion proteins. Titers were measured following 5 days of cultivation in culture tubes containing YPD 10 medium. HYP3, 1,8-cineole synthase from Hypoxylon sp. E7406B; AgGPPS2, GPP synthase from A. grandis; $\mathrm{P}_{\text {ANT, }}$. toruloides adenine nucleotide translocase promoter; GgFPS, FPP synthase from Gallus gallus

gallus (chicken). The best strain harboring $\mathrm{P}_{A N T}-H Y P 3$ and $\mathrm{P}_{T E F 1}-G g F P S(N 144 W), 345$, reached a 1,8-cineole titer of $143 \mathrm{mg} / \mathrm{L}$.
Additional strains were constructed to test further configurations of GgFPS(N144W) and HYP3 (Fig. 4c). Translational fusions of the two genes were expressed 
under control of $\mathrm{P}_{\text {GAPDH }}$ and although one configuration (strain 338, with GgFPS(N144W) N-terminal) was significantly better than the other (strain 340), the best 1,8-cineole titer was only $30 \%$ of that reached by strain 345 . When $\mathrm{P}_{T E F I^{-}}-G g F P S(N 144 W)$ was combined with HYP3 under control of $\mathrm{P}_{G A P D H}$ rather than $\mathrm{P}_{A N T}$ (strain 343) the 1,8-cineole titer was surprisingly much lower, compared to that of strain 345 . The combination of both $\mathrm{P}_{G A P D H^{-}} H Y P 3$ and $\mathrm{P}_{A N T}$ HYP3 with $\mathrm{P}_{\text {TEFI }}$-GgFPS(N144W) via gene stacking (strain 342) resulted in a 1,8-cineole titer matching that of 345 , indicating that perhaps a plateau had been reached that might only be surpassed by increasing flux through the mevalonate pathway.

\section{Targeted mevalonate pathway metabolic analysis}

Since this is a first attempt to engineer the mevalonate pathway in $R$. toruloides, we supplemented the global proteomic and transcriptomic data gathered from the $\alpha$-bisabolene strains with targeted metabolomic analysis of mevalonate pathway intermediates to inform an engineering strategy. Little is known about mevalonate pathway regulation in this species and, particularly as omics data showed that several pathway genes (ERG10, ERG13, ERG20) were upregulated in response to $\alpha$-bisabolene synthase overexpression (Fig. 2), it was considered prudent to avoid assumptions on rate limiting steps. At the same time, mevalonate pathway engineering strategies in S. cerevisiae and other fungi typically start with overexpression of 3-hydroxy-3-methyl-glutaryl-coenzyme A reductase (HMGR) and it was of interest to see how this would impact pathway metabolite levels [31-33]. Therefore, a new strain (312) to be included in the metabolomic analysis was constructed by the introduction of a truncated HMGR from Cricetulus griseus under control of the GAPDH promoter $\left(\mathrm{P}_{G A P D H^{-}}-t C g H M G R\right)$ into strain GB2. Mevalonate pathway metabolites were analyzed for four strains, $R$. toruloides WT, BIS3, GB2, and 312, which were grown for $40 \mathrm{~h}$ to late exponential phase in SD medium containing $20 \mathrm{~g} / \mathrm{L}$ glucose (Fig. 5). Intracellular levels of the early and late metabolites of the mevalonate pathway, acetyl-CoA and IPP/DMAPP, respectively, did not vary greatly between the $\alpha$-bisabolene-producing strains. However, the acetyl-CoA concentration was higher in the $\alpha$-bisabolene producing strains, compared to WT. Mevalonate accumulated in all strains, but was around $75 \%$ higher in strain GB2 and over fivefold higher in strain 312, compared to levels in $R$. toruloides WT. Mevalonate 5-phosphate did not accumulate in $R$. toruloides WT or BIS3 but was detected in GB2 and 312, with a sevenfold higher concentration in the latter strain. The approximate intracellular mevalonate concentration in strain $312(27 \mu \mathrm{M})$ was around 20-fold higher than acetyl-CoA $(1.6 \mu \mathrm{M})$ and mevalonate 5-phosphate $(1.1 \mu \mathrm{M})$.

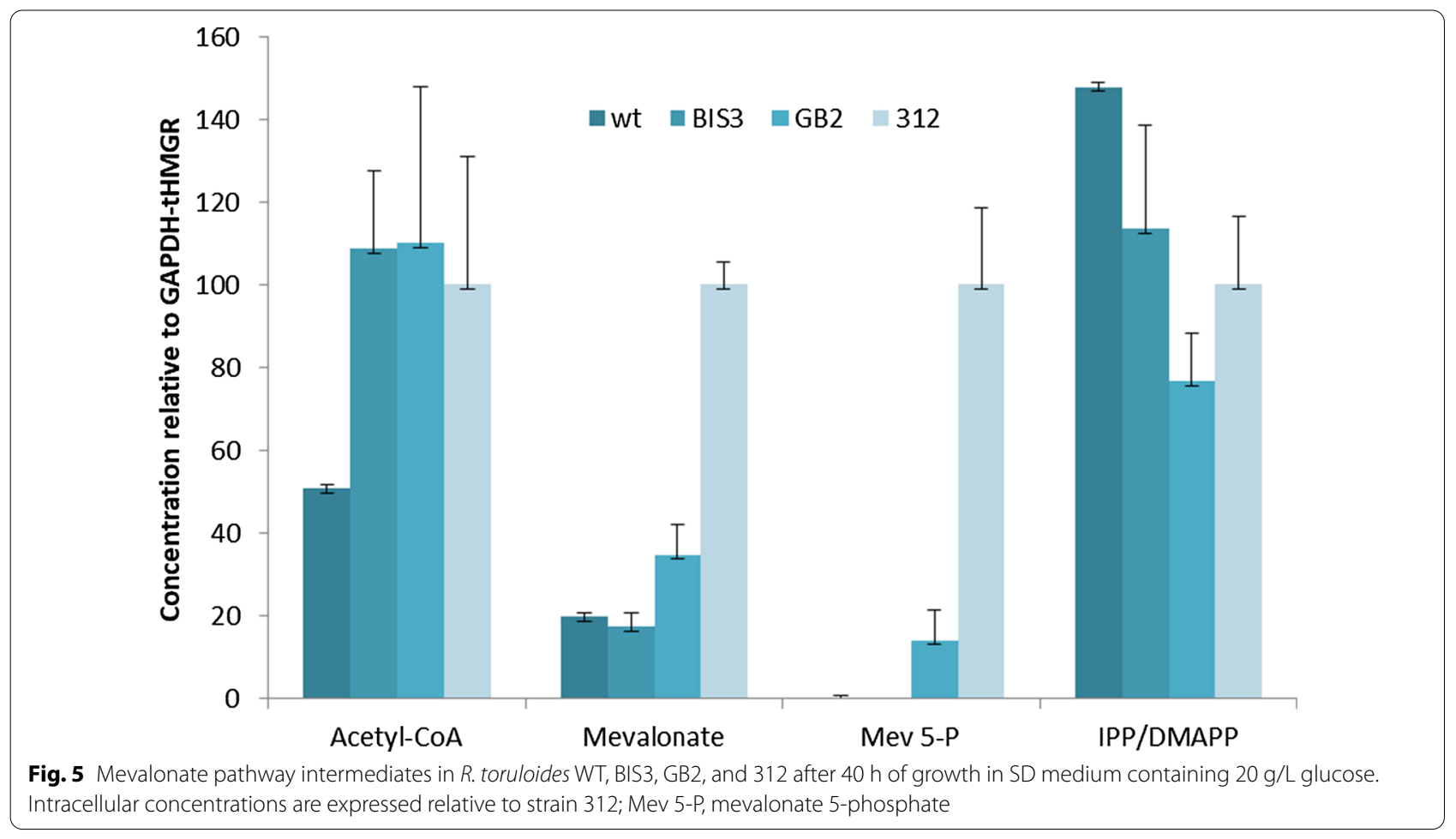


The data suggest that increasing production of $\alpha$-bisabolene through high-level expression of $A g B I S$ in strain GB2 promotes flux through the mevalonate pathway leading to an increase in mevalonate and mevalonate 5 -phosphate levels. Since overexpression of $t C g H M G R$ amplifies this effect, it seems likely that HMGR is somewhat rate limiting, as is the case in many other eukaryotes [31-33], but that production of mevalonate and mevalonate 5-phosphate emerge as downstream bottlenecks. Thus, HMGR and the two enzymes that process mevalonate and mevalonate 5-phosphate, MK and PMK, respectively, are possible overexpression targets for increasing mevalonate pathway flux in $R$. toruloides.

\section{Mevalonate pathway engineering}

Orthologs of the central mevalonate genes (encoding HMGR, MK, or PMK) were selected for expression in $R$. toruloides based on their characteristics or success in engineering terpene production in other organisms. The promoters, $\mathrm{P}_{A N T}, \mathrm{P}_{S K P 1}$ and $\mathrm{P}_{D U F}$ were selected for transgene expression based on previous characterization as very strong, strong, and medium strength (annotated as P15, P6, and P21, respectively, in that study) [15]. Prior to the combination of the candidate genes onto single constructs, they were tested individually for impact on $\alpha$-bisabolene and 1,8-cineole titers by expression in strains GB2 and 345, respectively. Two HMGR candidates, from Delftia acidovorans (DaHMGR) and Silicibacter pomeroyi (SpHMGR), were the most impactful on 1,8-cineole titers when expressed in strain 345 under control of the two strongest promoters, $\mathrm{P}_{A N T}$ and $\mathrm{P}_{\text {SKP1 }}$ (Additional file 1: Fig. S2a). SpHMGR was previously employed to engineer the mevalonate pathway in combination with central metabolism to increase terpene yields in S. cerevisiae [4] while DaHMGR was previously employed to engineer flux through a heterologous mevalonate pathway in E. coli [34]. Both are bacterial, $\mathrm{NADH}$-dependent HMGRs that lack the membranebound domains of eukaryotic HMGRs. Expression of heterologous MK and PMK genes, however, had a less marked impact on terpene production; perhaps not too surprising as the metabolic analysis suggests that they might only become limiting when HMGR is overexpressed (Fig. 5). Nevertheless, orthologs were down selected for expression in combinatorial constructs based on the available data. MKs, responsible for conversion of mevalonate to mevalonate-5-phosphate, are often subject to feedback inhibition by prenyl phosphates or mevalonate diphosphate but several Archaeal MKs are not subject to this control mechanism [35]. An Archaeal MK, from Methanosaeta concilii (McMK), which was previously found to be kinetically $\left(k_{\text {cat }} / \mathrm{K}_{\mathrm{m}}\right)$ more favorable than MKs from other Archaea and S. cerevisiae, had the most favorable impact on $\alpha$-bisabolene production in $R$. toruloides when under control of the medium-strong promoter, $\mathrm{P}_{S K P 1}$ (Additional file 1: Fig. S2b) [35]. Thus, $\mathrm{P}_{A N T}$ DaHMGR, $\mathrm{P}_{A N T}-S p H M G R$ and $\mathrm{P}_{S K P 1}-M c M K$ were selected for expression and the remaining promoter, $\mathrm{P}_{D U F}$, was selected to drive expression of PMKs from S. cerevisiae (ScPMK) and Streptococcus pneumoniae (SpPMK) (Additional file 1: Fig. S2c) [36, 37].

Various combinations of the selected HMGR, MK and PMK expression cassettes were assembled onto single plasmid backbones for testing in the $\alpha$-bisabolene and 1,8-cineole production strains, GB2 and 345 , respectively. For one configuration of these genes $\left(\mathrm{P}_{A N T}\right.$ SpHMGR, $\mathrm{P}_{S K P 1}$-McMK, and $\mathrm{P}_{D U F}$-SCPMK), three versions were built using different methods for codon optimization of the three coding sequences. Transformation of these constructs into the 1,8-cineole producing strain, 345, revealed that an in-house codon optimization method (expression cassette optimization, ECO), based on a combination of learnings from literature and analysis of $R$. toruloides RNAseq and proteomics data, worked poorly while the approaches of using the most preferred codon in $R$. toruloides for each amino acid (high-CAI, $\mathrm{HC}$ ) or Genscript's codon optimization algorithm (Genscript-optimized, GO) produced similar results (Fig. 6a). The GO method is also strongly biased towards selection of the most preferred codons and the output DNA sequences for the $\mathrm{HC}$ and GO methods differed by only a few percent. The success of the high-CAI codon optimization methods is surprising, but has been borne out by other studies in $R$. toruloides [38]. Four constructs that contain HMGR, MK and PMK coding sequences, optimized using the HC method, were transformed into strain 345 by ATMT and the resulting strains were screened for 1,8-cineole production. Constructs pGEN485 ( $\mathrm{P}_{A N T}$-SpHMGR, $\quad \mathrm{P}_{S K P 1}$-McMK, $\mathrm{P}_{D U F}$-SpPMK) and pGEN-486 ( $\mathrm{P}_{A N T}$-DaHMGR, $\mathrm{P}_{S K P 1}$-McMK, $\mathrm{P}_{D U F}$-ScPMK) were the most successful of these, resulting in 1,8-cineole titers of over $1 \mathrm{~g} / \mathrm{L}$ in strains 320 and 322 (Fig. 6b). The fact that similar titers were achieved with coupling of two different HMGR/PMK pairs suggests that pathway optimization may depend as much on achieving a balance between enzymes in the pathway as finding individual successful orthologs.

\section{Medium and process optimization}

To test whether terpene production may be limited by the lack of certain nutrients in corn stover hydrolysate, strain GB2 was grown in DMR-EH media supplemented individually with each of the components present in yeast nitrogen base (Difco). Of the supplements, thiamine hydrochloride, pyridoxine hydrochloride, $\mathrm{FeSO}_{4}$, and 

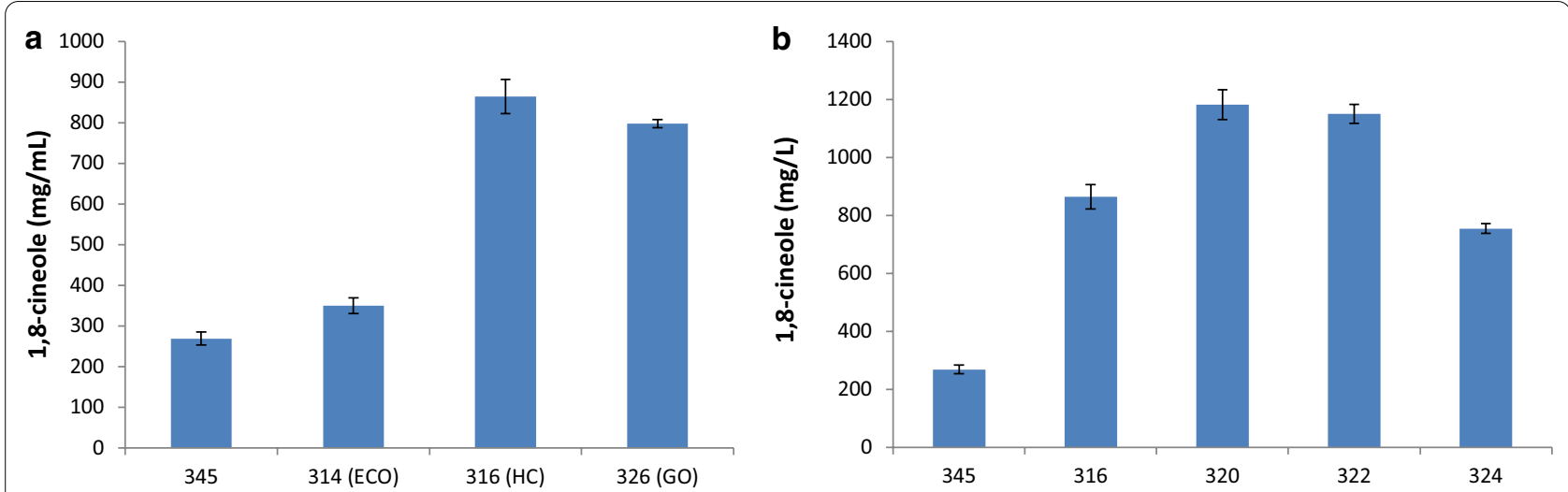

Fig. 6 Boosting production of 1,8-cineole in R. toruloides by overexpression of central mevalonate pathway enzymes, HMGR, MK, and PMK. a Comparison of three different methods for codon optimization ( $\mathrm{ECO}, \mathrm{HC}$, and $\mathrm{GO}$, described in Materials and Methods) for constructs harboring $P_{A N T}-S P H M G R, P_{S K P 1}-M C M K$, and $P_{D U F}-S C P M K$. b Various combinations of $P_{A N T}-H M G R, P_{S K P 1}-M K$, and $P_{D U F}-P M K$, described in Table 1, all codon-optimized using the $\mathrm{HC}$ method. Strains were cultivated in flower plates containing GXY medium and 1,8-cineole was measured at 7 days. HMGR, 3-hydroxy-3-methyl-glutaryl-coenzyme A reductase; MK, mevalonate kinase; PMK, phosphomevalonate kinase

$\mathrm{Na}_{2} \mathrm{SO}_{4}$ positively impacted production of $\alpha$-bisabolene (Additional file 1: Fig. S3).

Using microtiter plates with flower-shaped wells (flower plates) to enhance aeration, the highest titers were achieved in DMR-EH medium supplemented with $5 \mathrm{~g} / \mathrm{L}\left(\mathrm{NH}_{4}\right)_{2} \mathrm{SO}_{4}, 100 \mathrm{mM}$ potassium phosphate $(\mathrm{pH}$ 6.0), $400 \mu \mathrm{g} / \mathrm{L}$ thiamine hydrochloride, $400 \mu \mathrm{g} / \mathrm{L}$ pyridoxine hydrochloride, $100 \mu \mathrm{g} / \mathrm{L} \mathrm{FeSO}_{4}$, and $1 \mathrm{mM} \mathrm{Na}_{2} \mathrm{SO}_{4}$ (Fig. 7). Production of 1,8-cineole increased in both the 345 parent strain and the mevalonate-engineered strain 322 , when cultured in the supplemented medium, with the latter producing $1.4 \mathrm{~g} / \mathrm{L}$. An $\alpha$-bisabolene titer of
$2.2 \mathrm{~g} / \mathrm{L}$ was attained in strain GB2 and this improved to $2.6 \mathrm{~g} / \mathrm{L}$ in the mevalonate-engineered strain 319.

\section{Reexamination of mevalonate pathway intermediates}

Although the expression of HMGR, MK, and PMK orthologs in strain 345 yielded a fourfold increase in 1,8-cineole titer, transformation of the same constructs into GB2 had a more muted impact on the $\alpha$-bisabolene titer. Targeted analysis of mevalonate pathway intermediates was employed to probe this disparity in impact on sesquiterpene versus monoterpene titers (Additional file 1: Fig. S4). Mevalonate, mevalonate 5-phosphate and
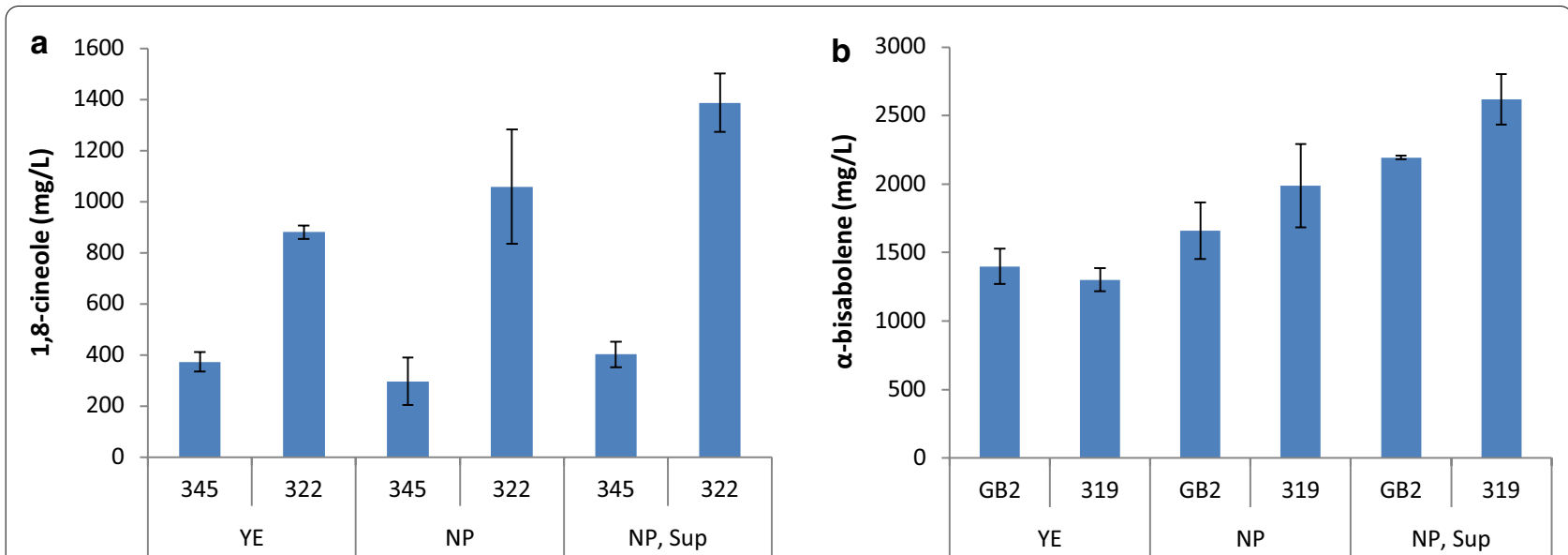

Fig. 7 Optimization of DMR-EH based media for the production of terpenes in $R$. toruloides. Strains were cultivated in flower plates and 1,8-cineole (a) and a-bisabolene (b) were quantified at 6 days and 12 days, respectively. YE, $10 \mathrm{~g} / \mathrm{L}$ yeast extract; NP, $5 \mathrm{~g} / \mathrm{L}$ ammonium sulfate and $100 \mathrm{mM}$ potassium phosphate (pH 6.0); Sup, supplementation with $400 \mu \mathrm{g} / \mathrm{L}$ thiamine hydrochloride, $400 \mathrm{\mu g} / \mathrm{L}$ pyridoxine hydrochloride, $100 \mu \mathrm{g} / \mathrm{L} \mathrm{FeSO}{ }_{4}$ and $1 \mathrm{mM} \mathrm{Na}_{2} \mathrm{SO}_{4}$. DMR-EH, Lignocellulosic hydrolysate prepared from corn stover by deacetylation and mechanical refining followed by enzymatic hydrolysis 
mevalonate 5-diphosphate levels were all higher in the $\alpha$-bisabolene producing parent strain, GB2, compared to the 1,8-cineole producing parent strain, 345. Upon overexpression of HMGR, MK, and PMK in these strains, intracellular levels of the same three pathway intermediates increased in both cases. However, higher levels were reached in the $\alpha$-bisabolene producing strain, 319, compared to the 1,8-cineole producing strain, 317. As the increase in mevalonate pathway flux translates into a larger increase in monoterpene than sesquiterpene titers, it is possible that the discrepancy is related to the balance between the $\mathrm{C}_{5}$ precursors IPP and DMAPP. Production of the monoterpene precursor GPP requires a 1:1 ratio of IPP to DMAPP, while the sesquiterpene precursor FPP requires a 2:1 ratio, suggesting IPP/DMAPP isomerase (IDI) is a possible engineering target. Overexpression of IDI has proved effective in increasing titers of sesquiterpenes and carotenoids in other organisms, with data suggesting that it becomes more important as higher IPP/DMAPP ratios are required for production of larger terpenes $[39,40]$. It is also possible that sesquiterpene production is limited by FPP synthase which, unlike GPP synthase in the 1,8-cineole producing strains, has not been overexpressed in the $\alpha$-bisabolene strains. However, since the quantity of $\alpha$-bisabolene produced in $R$. toruloides is twice that for 1,8-cineole, it is entirely possible that both terpenes are limited by a common factor, such as mevalonate diphosphate decarboxylase or by a nutrient in the DMR-EH medium. These will be among the factors to be evaluated in future engineering studies. A summary of key strains and metabolic pathway changes are shown in Additional file 1: Table S3 and Figure S5, respectively.

\section{Conclusions}

The $\alpha$-bisabolene and 1,8-cineole titers attained in $R$. toruloides, 2.6 and $1.4 \mathrm{~g} / \mathrm{L}$, respectively, appear to be the highest titers achieved for these terpenes in a microbial host to date. Importantly, they are achieved by growth on corn stover hydrolysate, supplemented only with $5 \mathrm{~g} / \mathrm{L}$ ammonium sulfate and a few other nutrients. The DMR-EH medium contains approximately $115 \mathrm{~g} / \mathrm{L}$ sugars (73 g/L glucose, $39 \mathrm{~g} / \mathrm{L}$ xylose and $3 \mathrm{~g} / \mathrm{L}$ arabinose) and the current yields for $\alpha$-bisabolene and 1,8-cineole $(0.022$ and $0.012 \mathrm{~g}$ terpene/g sugars, or about $10 \%$ and $5 \%$ of the maximum biochemical yields via the mevalonate pathway, respectively) leave substantial room for improvement. A large amount of carbon is usually directed towards lipid synthesis in $R$. toruloides and then later recycled back to acetyl-CoA, as needed. This introduces a large degree of inefficiency to the terpene biosynthetic pathway, significantly reducing the maximum biochemical yield. Therefore, reduction of flux from acetyl-CoA to lipids remains a high priority for future work in this host, a feat that will require further development of genetic tools for downregulation of gene expression.

\section{Materials and methods \\ Media}

YPD medium (10 g/L yeast extract, $20 \mathrm{~g} / \mathrm{L}$ peptone, and $20 \mathrm{~g} / \mathrm{L}$ glucose), prepared using components from Difco (Becton, Dickinson and Company (BD), Franklin Lakes, NJ, USA), and lysogeny broth (LB, Miller formulation, VWR, Radnor, PA, USA) were used for routine growth of $R$. toruloides. YPD 10 refers to YPD containing $100 \mathrm{~g} / \mathrm{L}$ glucose. GXY medium contained $75 \mathrm{~g} / \mathrm{L}$ glucose, $25 \mathrm{~g} / \mathrm{L}$ xylose and $10 \mathrm{~g} / \mathrm{L}$ yeast extract (Difco). Synthetic defined (SD) medium was prepared following manufacturers' instructions, using Difco yeast nitrogen base (YNB) without amino acids (Becton, Dickinson \& Co., Sparks, MD, USA) and complete supplement mixture (CSM; Sunrise Science Products, San Diego, CA, USA). Lignocellulosic hydrolysate was prepared from corn stover by deacetylation and mechanical refining followed by enzymatic hydrolysis, as described previously, and is referred to here as DMR-EH [13]. DMR-EH, supplied by the National Renewable Energy Laboratory (NREL), was centrifuged to remove solids and then sterilized by passage through a $0.2-\mu \mathrm{M}$ filter before use. Strains were normally grown at $30{ }^{\circ} \mathrm{C}$ on solid medium containing $15 \mathrm{~g} / \mathrm{L}$ agar, or in liquid medium with shaking at $200 \mathrm{rpm}$ (Adolf Kühner AG, SBM-X, Birsfelden, Switzerland) unless strains were grown in plate format, in which case higher shaking speeds were employed (see Terpene production and quantitation, below).

\section{Strains, plasmids and transformation}

R. toruloides IFO0880 and Agrobacterium tumefaciens EHA 105 were obtained from Jeffrey Skerker at UC Berkeley [14]. Binary plasmids for heterologous gene expression were built by GenScript (Piscataway, NJ, USA). Heterologous DNA was introduced into $R$. toruloides by Agrobacterium tumefaciens-mediated transformation (ATMT) as described previously [2] or in some cases by chemical transformation using lithium acetate [15]. In both cases, transformation results in integration of the heterologous DNA at random loci with variable copy numbers. Strains and plasmids used in this study can be found in Table 1 and are available upon request; more details, including plasmid maps, can be found on the Agile BioFoundry Strain Registry (http://public-regis try.agilebiofoundry.org/ [16]).

\section{Codon optimization}

Genes encoding terpene synthases and prenyl transferases were codon-optimized for $R$. toruloides by 
Table 1 Overview of constructs used to engineer terpene production in R. toruloides

\begin{tabular}{|c|c|c|}
\hline Genotype/features & Name & Registry ID \\
\hline \multicolumn{3}{|l|}{ Plasmids } \\
\hline$P_{G A P D H}-B I S-T_{N O S}, N A T$ & GPD-BIS-NOS & JPUB_017422 \\
\hline $\mathrm{P}_{\text {ANT }}-\mathrm{BIS}^{-\mathrm{T}_{N O S}}, \mathrm{HYG}$ & pGEN-295 & JPUB_017423 \\
\hline $\mathrm{P}_{\text {ANT }}-\mathrm{HYP3}-\mathrm{T}_{N O S}, \mathrm{HYG}$ & pGEN-299 & ABFPUB_000356 \\
\hline 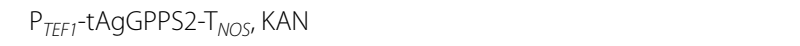 & pGEN-376 & ABFPUB_000335 \\
\hline $\mathrm{P}_{\text {ANT }}-\mathrm{HYP3} 3-\mathrm{T}_{\text {NOS }}, \mathrm{P}_{\text {TEF } \mathrm{T}}-\mathrm{AgGPPS} 2-\mathrm{T}_{355}, \mathrm{KAN}$ & pGEN-308 & ABFPUB_000348 \\
\hline $\mathrm{P}_{\text {GAPDH }}-\mathrm{HYP3}^{-\mathrm{T}_{N O S}}{ }_{\mathrm{N}} \mathrm{NAT}$ & pGEN-300 & ABFPUB_000357 \\
\hline $\mathrm{P}_{\text {TEF }}-\mathrm{AgGPPS} 2-\mathrm{T}_{355}, \mathrm{BLE}$ & pGEN-305 & ABFPUB_000351 \\
\hline 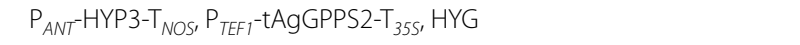 & pGEN-309 & ABFPUB_000353 \\
\hline $\mathrm{P}_{\text {TEF }}-$ RtERG20(F91W)-T ${ }_{N O S}$, KAN & pGEN-374 & ABFPUB_000331 \\
\hline $\mathrm{P}_{\text {TEF } 1}-\mathrm{RtERG} 20(\mathrm{~F} 91 \mathrm{~W}, \mathrm{~N} 122 \mathrm{~W})-\mathrm{T}_{\text {NOS }}, \mathrm{KAN}$ & pGEN-375 & ABFPUB_000333 \\
\hline 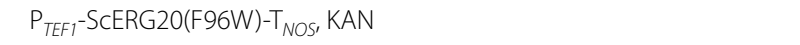 & pGEN-372 & ABFPUB_000327 \\
\hline $\mathrm{P}_{\text {TEF } 1}$-SCERG20(F96W, N127W)-T ${ }_{\text {NOS }}, \mathrm{KAN}$ & pGEN-373 & ABFPUB_000329 \\
\hline $\mathrm{P}_{\text {ANT }}-\mathrm{HYP} 3-\mathrm{T}_{N O S}, \mathrm{P}_{\text {TEF }}-\mathrm{GgFPS}(\mathrm{N} 144 \mathrm{~W})-\mathrm{T}_{355}, \mathrm{NAT}$ & pGEN-307 & ABFPUB_000344 \\
\hline$P_{G A P D H}-H Y P 3-G g F P S(N 144 W)-T_{N O S}$ (fusion), NAT & pGEN-379 & ABFPUB_000339 \\
\hline$P_{G A P D H}-G g F P S(N 144 W)-H Y P 3-T_{N O S}$ (fusion), NAT & pGEN-378 & ABFPUB_000337 \\
\hline$P_{T E F 1}-G g F P S(N 144 W)-T_{N O S}, K A N$ & pGEN-304 & ABFPUB_000341 \\
\hline$P_{G A P D H}-{ }^{-t C g H M G R-T}{ }_{N O S}, K A N$ & GPD-tHMGR & ABFPUB_000355 \\
\hline$P_{A N T}-S p H M G R-T_{N O S}, P_{S K P 1}-M c M K-T_{S K P I}, P_{D U F}-S C P M K-T_{D U F}(E C O), K A N$ & pGEN-446 & ABFPUB_000313 \\
\hline$P_{A N T}-S p H M G R-T_{N O S}, P_{S K P T}-M_{C M K}-T_{S K P 1}, P_{D U F}-S C P M K-T_{D U F}(H C), K A N$ & pGEN-484 & ABFPUB_000315 \\
\hline$P_{A N T}-S p H M G R-T_{N O S}, P_{S K P 1}-M C M K-T_{S K P 1}, P_{D U F}-S C P M K-T_{D U F}(G O), K A N$ & pGEN-494 & ABFPUB_000325 \\
\hline$P_{A N T}-S p H M G R-T_{N O S}, P_{S K P T}-M c M K-T_{S K P 1}, P_{D U F}-S p P M K-T_{D U F}(H C), K A N$ & pGEN-485 & ABFPUB_000318 \\
\hline$P_{A N T}-$ DaHMGR-T ${ }_{N O S}, P_{S K P 1}-M_{C M K}-T_{S K P 1}, P_{D U F}-S C P M K-T_{D U F}(H C), K A N$ & pGEN-486 & ABFPUB_000321 \\
\hline$P_{A N T}-$ DaHMGR-T ${ }_{N O S}, P_{S K P T}-M_{C M K-T}{ }_{S K P}, P_{D U F}-S p P M K-T_{D U F}(H C), K A N$ & pGEN-487 & ABFPUB_000323 \\
\hline \multicolumn{3}{|l|}{ Strains } \\
\hline R. toruloides IFO0880, mating type A2 (WT) & & ABFPUB_000014 \\
\hline 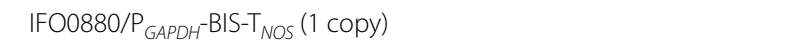 & BIS18 & JPUB_013664 \\
\hline IFO0880/P ${ }_{\text {GAPDH }}{ }^{-B I S-T_{N O S}}$ (7 copies) & BIS14 & JPUB_017424 \\
\hline IFO0880/P ${ }_{\text {GAPDH }}-\mathrm{BIS}-\mathrm{T}_{\text {NOS }}(10$ copies $)$ & $\mathrm{BIS} 3$ & JPUB_009679 \\
\hline 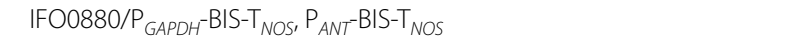 & GB2 & ABFPUB_000311 \\
\hline 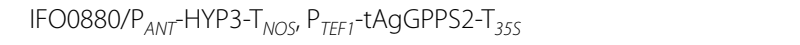 & 336 & ABFPUB_000336 \\
\hline 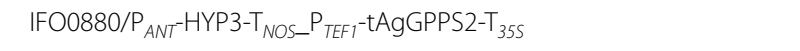 & 350 & ABFPUB_000350 \\
\hline 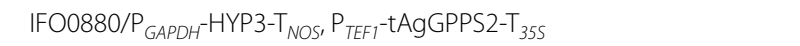 & 352 & ABFPUB_000352 \\
\hline 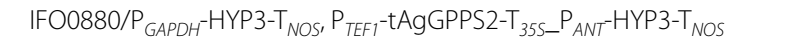 & 354 & ABFPUB_000354 \\
\hline 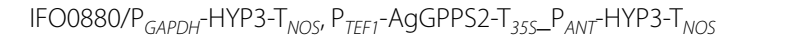 & 349 & ABFPUB_000349 \\
\hline 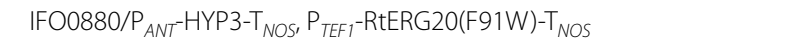 & 332 & ABFPUB_000332 \\
\hline 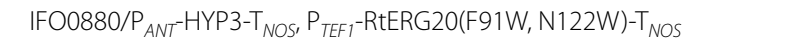 & 334 & ABFPUB_000334 \\
\hline 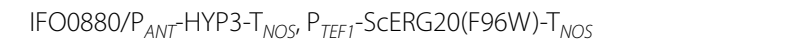 & 328 & ABFPUB_000328 \\
\hline 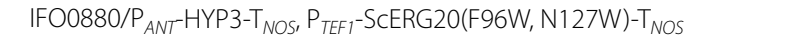 & 330 & ABFPUB_000330 \\
\hline IFO0880/P $\mathrm{P}_{\text {ANT }}-\mathrm{HYP} 3-\mathrm{T}_{\text {NOS_}} \mathrm{P}_{\text {TEF1 } 1}-\mathrm{GgFPS}(\mathrm{N} 144 \mathrm{~W})-\mathrm{T}_{355}$ & 345 & ABFPUB_000345 \\
\hline IFO0880/P GAPDH - HYP3-GgFPS(N144W)-T ${ }_{N O S}$ (fusion) & 340 & ABFPUB_000340 \\
\hline IFO0880/P GAPDH - GgFPS(N144W)-HYP3-T ${ }_{\text {NOS }}$ (fusion) & 338 & ABFPUB_000338 \\
\hline IFO0880/P GAPDH - HYP3-T NOS, $\mathrm{P}_{\text {TEF } 1}-\mathrm{GgFPS}(\mathrm{N} 144 \mathrm{~W})-\mathrm{T}_{\text {NOS }}$ & 343 & ABFPUB_000343 \\
\hline 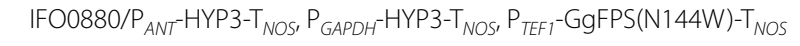 & 342 & ABFPUB_000342 \\
\hline IFO0880/P $\mathrm{P}_{\text {GAPDH }}-\mathrm{BIS}-\mathrm{T}_{\text {NOS }}, \mathrm{P}_{\text {ANT }}-\mathrm{BIS}-\mathrm{T}_{\text {NOS }}, \mathrm{P}_{\text {GAPDH }}-\mathrm{tCgHMGR-T_{NOS }}$ & 312 & ABFPUB_000312 \\
\hline $345 / P_{A N T}-S p H M G R-T_{N O S-} P_{S K P 1}-M_{C M K-T}{ }_{S K P 1} P_{D U F}-S C P M K-T_{D U F}(E C O)$ & 314 & ABFPUB_000314 \\
\hline 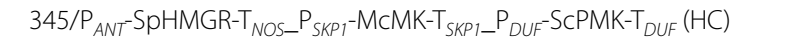 & 316 & ABFPUB_000316 \\
\hline $345 / P_{\text {ANT }}-S p H M G R-T_{\text {NOS_ }} P_{S K P 1}-M_{C M K-T}{ }_{S K P 1} P_{D U F}-S C P M K-T_{D U F}(G O)$ & 326 & ABFPUB_000326 \\
\hline $345 / P_{A N T}-S p H M G R-T_{\text {NOS }}-P_{S K P 1}-M_{C M K}-T_{S K P 1} P_{D U F}-S p P M K-T_{D U F}(H C)$ & 320 & ABFPUB_000320 \\
\hline
\end{tabular}


Table 1 (continued)

\begin{tabular}{|c|c|c|}
\hline Genotype/features & Name & Registry ID \\
\hline 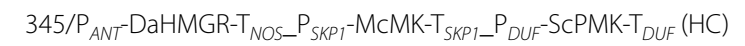 & 322 & ABFPUB_000322 \\
\hline 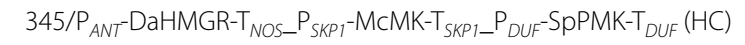 & 324 & ABFPUB_000324 \\
\hline $345 / P_{A N T}-S p H M G R-T_{N O S-} P_{S K P 1}-M C M K-T_{S K P 1-} P_{D U F}-S C P M K-T_{D U F}(H C)$ & 317 & ABFPUB_000317 \\
\hline$G B 2 / P_{A N T}-S p H M G R-T_{N O S-} P_{S K P 1}-M C M K-T_{S K P I-} P_{D U F}-S p P M K-T_{D U F}(H C)$ & 319 & ABFPUB_000319 \\
\hline
\end{tabular}

Strains and plasmids used in this study are available upon request through strain registries of the Agile BioFoundry (http://public-registry.agilebiofoundry.org/) and the Joint BioEnergy Institute (https://public-registry.jbei.org/), designated by ABF and JBx, respectively [16]. Unless indicated, sequences are from $R$. toruloides, with the exception of antibiotic resistance genes. GAPDH, glyceraldehyde 3-phosphate dehydrogenase; BIS, a-bisabolene synthase from Abies grandis (NCBI Accession Number, 081086), NOS, nopaline synthase from A. tumefaciens (MK078637); ANT, adenine nucleotide translocase; TEF1, translational elongation factor; NAT, nourseothricin resistance cassette; HYG, Hygromycin B resistance cassette; KAN, kanamycin (G418) resistance cassette; HYP3, 1,8-cineole synthase from Hypoxylon sp. E7406B (AHY23922); AgGPPS2, GPP synthase from A. grandis (AAN01134); tAgGPPS2, AgGPPS2 with the N-terminal plastid transit peptide (84 amino acids) removed; 35S, 35 S mRNA gene from cauliflower mosaic virus (KJ716236); RtERG20, FPP synthase from R. toruloides (PRQ75922); ScERG20, FPP synthase from S. cerevisiae (NP_012368); GgFPS, FPP synthase from Gallus gallus (P08836.2); tCgHMGR, HMGR from Cricetulus griseus, truncated by removal of $351 \mathrm{~N}$-terminal amino acids (XP 027257481), SpHMGR, HMGR from Silicibacter pomeroyi (WP 011241944); SKP1, S-phase kinase-associated protein 1 from R. toruloides (PRQ77980); McMK, MK from Methanosaeta concilii (WP_013720012); DUF, domain of unknown function from R. toruloides (PRQ75822); ScPMK, PMK from S. cerevisiae (AJS65138); SpPMK, PMK from Streptococcus pneumoniae (WP_044791288); and DaHMGR, HMGR from Delftia acidovorans (WP_099752490). Codon optimization was performed by Genscript using a R. toruloides codon table, except where indicated by HC (high-CAl method) and ECO (expression cassette optimization). In the strain genotype description, a comma indicates sequential insertion (stacking) while an underscore indicates that the cassettes are on the same construct

GenScript, based on a custom $R$. toruloides IFO0880 codon usage table (https://mycocosm.jgi.doe.gov/Rhoto_ IFO0880_4/Rhoto_IFO0880_4.home.html). Genes encoding central mevalonate pathway enzymes were codon-optimized by GenScript, as above, and also by two other methods. The first of these (high codon adaptation index (high-CAI); HC) used the same codon usage table and Codon Optimizer software (genomes.urv.es/ OPTIMIZER/) to select the codon most-utilized by $R$. toruloides for each amino acid. The second method (Expression Cassette Optimization, ECO) employed a balanced approach in which the second codon was optimized to approximate an optimal translational start consensus; less frequently used codons were selected for amino acids 3-10 in order to reduce mRNA secondary structure around the start codon; codons 11 to 45 and the last 50 codons were high-CAI and the remaining codons were selected to match the average usage in $R$. toruloides, using the same codon table and Codon Optimizer software [17-19].

\section{Terpene production and quantitation}

Typically, $R$. toruloides strains were initially inoculated from YPD agar plates into LB medium and grown overnight to mid-log phase at $30{ }^{\circ} \mathrm{C}$ with shaking at $200 \mathrm{rpm}$. Optical density (OD) was determined by measuring absorbance at $600 \mathrm{~nm}\left(\mathrm{OD}_{600}\right)$ using a standard cuvette (10 mm lightpath) in a SpectraMax Plus 384 Microplate Reader (Molecular Devices, San Jose, CA, USA). For measurement of terpene production, strains were subcultured from the LB preculture into $5 \mathrm{~mL}$ production medium (YPD, YPD 10 , GXY, or DMR-EH) in $20 \mathrm{~mm}$ diameter culture tubes at a starting $\mathrm{OD}_{600}$ of 0.1 . A $20 \%$ $(\mathrm{v} / \mathrm{v})$ dodecane (Sigma-Aldrich, St. Louis, MO, USA) overlay $(1 \mathrm{~mL})$ was added to capture terpenes produced during growth at $30{ }^{\circ} \mathrm{C}$ with shaking at $200 \mathrm{rpm}$. In some cases the dodecane overlay was spiked with an internal standard to adjust for evaporation-for production of 1,8-cineole, the dodecane overlay contained 1,4-cineole (Sigma-Aldrich) as an internal standard, while pentadecane (Sigma-Aldrich) was used as an internal standard for cultures producing $\alpha$-bisabolene.

In some cases, growth and terpene production was carried out in 48-well flower plates (M2P-48-B, m2p-labs, Islandia, NY, USA) sealed with an Aeraseal air-permeable seal (Excel Scientific, Victorville, CA, USA), essentially as described above but employing a 1-mL culture volume and incubation at $30{ }^{\circ} \mathrm{C}, 1,000 \mathrm{rpm}$, and $70 \%$ relative humidity on a Multitron II platform shaker (Infors HT, Annapolis Junction, MD, USA).

For determination of 1,8-cineole product titers, the dodecane overlay was sampled and diluted in ethyl acetate spiked with $40 \mu \mathrm{g} / \mathrm{mL}$ pentadecane (to normalize for injection volume and instrument response during quantitation) followed by analysis by gas chromatography-mass spectrometry (GC-MS). For quantitation of $\alpha$-bisabolene, the dodecane overlay was diluted into dodecane containing $40 \mu \mathrm{g} / \mathrm{mL}$ hexadecane (SigmaAldrich). GC-MS analysis was performed on an Agilent GC-MS 6890-5973 system (Agilent Technologies, Inc., Santa Clara, CA, USA) equipped with an Agilent DB- $5 \mathrm{~ms}$ capillary column (30 m length, $0.25 \mathrm{~mm}$ diameter, $0.25 \mu \mathrm{m}$ film thicknesses).

The separation and detection method for 1,8-cineole was as follows: $1 \mu \mathrm{L}$ of sample was injected into the GC inlet in splitless mode with the GC oven temperature held at $80{ }^{\circ} \mathrm{C}$ for $1 \mathrm{~min}$, ramped to $150{ }^{\circ} \mathrm{C}$ at $20^{\circ} \mathrm{C} /$ min, and then ramped to $300{ }^{\circ} \mathrm{C}$ at $40{ }^{\circ} \mathrm{C} / \mathrm{min}$. The inlet 
purge flow rate was set to $11 \mathrm{~mL} / \mathrm{min}$ at $0 \mathrm{~min}$. The mass spectrometer was timed to turn off during elution of the solvents, ethyl acetate ( 0 to $2.5 \mathrm{~min}$ ) and dodecane (4 to $5.8 \mathrm{~min})$. The mass spectrometer was set to selected ion mode (SIM), targeting ions representative of 1,8-cineole and the internal standards 1,4-cineole and pentadecane, with mass/charge $(\mathrm{m} / \mathrm{z})$ ratios of $70,85,93,136,139$, and 154. 1,8-cineole concentrations were calculated using an authentic 1,8-cineole (Sigma-Aldrich) standard curve after normalization of peak areas to those of the pentadecane internal standard. Evaporation of the 1,8-cineole product during production was corrected by quantitation of the 1,4-cineole that was spiked into the dodecane overlay at the outset of cell culture. The separation method for $\alpha$-bisabolene [20] was performed as described previously, using an $\alpha$-bisabolene standard prepared in-house. $\alpha$-Bisabolene was quantified after normalization of peak areas to those of the hexadecane internal standard. In cases where the aeration rate was high during growth (e.g., during cultivation in a bioreactor), evaporation was corrected by quantification of the pentadecane that was spiked into the dodecane overlay at the outset of cell culture.

\section{Genome sequencing and determination of transgene copy number}

Copy number of the integrated $\alpha$-bisabolene synthase gene was determined from whole-genome resequencing of transformants. Whole-genome resequencing was performed at the Joint Genome Institute (JGI) using Illumina paired-end sequencing method using MiSeq $2 \times 150$ bp (JGI Proposal ID 503,063 and 1889). The sequence data have been deposited with the NCBI BioProject database under the following accession numbers: PRJNA370932 (BIS1), PRJNA370806 (BIS2), PRJNA370807 (BIS3), PRJNA370808 (BIS4), PRJNA370809 (BIS5), PRJNA370810 (BIS6), PRJNA370811 (BIS7), PRJNA370812 (BIS8), PRJNA370813 (BIS9), PRJNA370814 (BIS10), PRJNA370815 (BIS11), PRJNA370816 (BIS12), PRJNA370817 (BIS13), PRJNA370818 (BIS14), PRJNA370819 (BIS15), PRJNA371224 (BIS16), PRJNA370821 (BIS17), PRJNA370822 (BIS18), PRJNA370823 (BIS19), PRJNA371225 (BIS20), and PRJNA441663 (GB2).

The sequenced reads were mapped using BWA-MEM [21] to the reference genome sequence of $R$. toruloides IFO0880 (https://mycocosm.jgi.doe.gov/Rhoto_IFO08 80_4/Rhoto_IFO0880_4.home.html) augmented with the sequence of the transformation plasmid containing $\mathrm{P}_{G A P D H^{-}}$BIS or $\mathrm{P}_{A N T}-\mathrm{BIS}$. The mapped reads were then sorted using SAMtools [22] and duplicate reads were marked using Picard Toolkit (Broad Institute, http:// broadinstitute.github.io/picard) to generate the BAM files. The copy number of $\mathrm{P}_{G A P D H^{-}}$BIS or $\mathrm{P}_{A N T}$ BIS was determined using CNVnator [23]. Bin sizes of 100, 200, and 1,000 bp were used for counting mapped reads within each bin and partitioning the read depth signal into segments. The average and standard deviation of read depth signal was evaluated for bin sizes of 100 and $200 \mathrm{bp}$, and copy number genotype was determined for the $\mathrm{P}_{G A P D H^{-}} \mathrm{BIS}$ or $\mathrm{P}_{A N T}$ - $\mathrm{BIS}$ region using each bin size. Estimated copy numbers were similar for bin sizes of 100 and $200 \mathrm{bps}$, and the results using $200 \mathrm{bp}$ bin were used in Fig. 1.

\section{Bioreactor-scale cultivation for a-bisabolene production}

Bioreactor cultivation was performed at the Advanced Biofuels Product Demonstration Unit (ABPDU) in a 2-L bioreactor (BIOSTAT B, Sartorius AG, Goettingen, Germany) containing $900 \mathrm{~mL}$ 75\% DMR-EH supplemented with $10 \mathrm{~g} / \mathrm{L}$ yeast extract (Difco) or defined nitrogen, as described in Results. A $20 \%(200 \mathrm{~mL})$ dodecane overlay $(\mathrm{v} / \mathrm{v})$, spiked with $200 \mathrm{mg} / \mathrm{L}$ pentadecane as an internal standard, was added to the bioreactor to capture $\alpha$-bisabolene produced during fermentation. The BIOSTAT $B^{\circledR}$ fermentation system (Sartorius AG) was employed in batch mode, using a jacketed 2-L borosilicate glass vessel (UniVessel ${ }^{\circledR}$, Sartorius AG) equipped with two 6-blade Rushton impellers, a dissolved oxygen (DO) probe (VisiFerm DO225, Hamilton Bonaduz AG, Bonaduz, Switzerland), and a pH probe (EasyFerm Plus VP 225, Hamilton Bonaduz AG). The bioreactor was inoculated to an $\mathrm{OD}_{600}$ of 1 from a culture propagated to mid-log phase on the same medium in $250-\mathrm{mL}$ shake flasks (approx. $100 \mathrm{~mL}$ ). Fermentation was carried out at $30{ }^{\circ} \mathrm{C}$ with air supplied at a sparge rate of $0.5 \mathrm{lpm}$ $(0.5 \mathrm{vvm})$ and constant agitation at $400 \mathrm{rpm}$. After initial adjustment of the growth medium $\mathrm{pH}$ to 6.0 , the $\mathrm{pH}$ was maintained at or above 5.0 using $2 \mathrm{~N}$ sodium hydroxide. Process values were monitored and recorded using the integrated Sartorius data acquisition software (BioPAT MFCS/win). Sugar consumption, OD, and $\alpha$-bisabolene production were measured over a period of 12 days.

\section{Quantification of xylose and glucose}

Prior to analysis, samples were diluted as appropriate and filtered through $0.45 \mu \mathrm{m}$ filters (VWR Centrifugal Filters) by centrifugation at $3000 \times \mathrm{g}$ for $3 \mathrm{~min}$. Sugars were quantified on a Dionex Ultimate 3000 system UHPLC (Agilent Technologies) using an Aminex HPX-87H column (BioRad, Hercules, CA, USA) and Thermo Scientific ${ }^{\mathrm{TM}}$ RefractoMax 520 Refractive Index Detector (RID) (Thermo Fisher Scientific, Waltham, MA, USA) as described previously [5]. 


\section{Transcriptomics and proteomics analysis}

Strains were cultivated in SD medium containing 1\% $(10 \mathrm{~g} / \mathrm{L})$ glucose. Regular samples were taken for measurement of growth and $\alpha$-bisabolene titer, and the samples taken at 18 and $48 \mathrm{~h}$ post-inoculation were selected for transcriptomics and proteomics analysis, representing the exponential and stationary phases of growth. For transcriptomics analysis, 1-mL samples were centrifuged and cells were washed three times with DEPCtreated water before flash freezing in liquid nitrogen. For proteomics analysis, cells were washed three times with $100 \mathrm{mM}$ ammonium bicarbonate and flash frozen in liquid nitrogen until usage.

Total RNA was isolated by disrupting harvested cells using an MP Biomedicals FastPrep bead beater (MP Biomedicals, Irvine, CA, USA) followed by purification using the QIAGEN RNeasy Mini kit with on-column DNase treatment following the manufacturer's protocol. $10 \mu \mathrm{g}$ of RNA was then used to prepare a sequencing library with the TruSeq RNA Library Preparation Kit v2 (Illumina, San Diego, CA, USA). The quality and concentrations of RNA samples and sequencing libraries were measured with an Agilent Bioanalyzer 2100 and Qubit. In addition, a q-PCR quantification of sequencing libraries was run using a KAPA library quantification kit (Roche, Pleasanton, CA, USA). RNA-sequencing was performed at the Agile BioFoundry using Illumina paired-end sequencing method using MiSeq $2 \times 75 \mathrm{bp}$. The sequence data have been deposited with the NCBI GEO database under accession number GSE156051 (Token for reviewers to access the data: krmpyckyhxqdfop). Sequenced RNA reads were trimmed and filtered using BBTools (https://jgi.doe.gov/data-and-tools/bbtoo ls/), and mapped to the reference genome sequence of $R$. toruloides IFO0880 augmented with the coding sequence of BIS using HISAT2 [24], Mapped reads were assigned to genes using featureCounts [25], and read counts were used to calculate Fragments Per Kilobase of transcript per Million mapped reads (FPKM).

For global proteomics analysis, to extract the protein from the cell pellets for global proteomics analysis, $1 \mathrm{~mL}$ of a mixture of chloroform:methanol (prepared 2:1 (v/v)) was pipetted into chloroform compatible $2 \mathrm{~mL}$ Sorenson MulTi $^{\mathrm{TM}}$ SafeSeal $^{\mathrm{TM}}$ microcentrifuge tubes (Sorenson bioscience, Salt Lake City, UT, USA) kept inside an ice block. Cell pellets were resuspended in $200 \mu \mathrm{L}$ of water and immediately transferred into the microcentrifuge tubes. Samples were vigorously vortexed, placed in an ice block for $10 \mathrm{~min}$, vortexed again for $20 \mathrm{~s}$ and centrifuged at $17,000 \times g$ for $10 \mathrm{~min}$ at $4{ }^{\circ} \mathrm{C}$. The upper and lower phases were removed. The remaining protein interphase was washed with methanol, vortexed and centrifuged at $17,000 \times g$ for
5 min to pellet the protein. The methanol supernatant was decanted into waste and the pellet lightly dried in a fume hood and then stored at $-80{ }^{\circ} \mathrm{C}$ until protein digestion. The protein interlayer pellet was digested by adding $200 \mu \mathrm{L}$ of an $8 \mathrm{M}$ urea solution to the protein pellets and vortexed into solution. A bicinchoninic acid (BCA) assay (Thermo Fisher Scientific) was performed to determine protein concentration. Following the assay, $10 \mathrm{mM}$ dithiothreitol (DTT) was added to the samples and incubated at $60{ }^{\circ} \mathrm{C}$ for $30 \mathrm{~min}$ with constant shaking at $800 \mathrm{rpm}$. Reduced cysteine residues were alkylated by adding $400 \mathrm{mM}$ iodoacetamide (Sigma-Aldrich) to a final concentration of $40 \mathrm{mM}$ and incubating in the dark at room temperature for $1 \mathrm{~h}$. Samples were then diluted eightfold for preparation for digestion with $100 \mathrm{mM} \mathrm{NH}_{4} \mathrm{HCO}_{3}, 1 \mathrm{mM} \mathrm{CaCl}$ and sequencing-grade modified porcine trypsin (Promega, Madison, WI, USA) was added to all protein samples at a 1:50 (w/w) trypsin-to-protein ratio for $3 \mathrm{~h}$ at $37{ }^{\circ} \mathrm{C}$. Digested samples were desalted using a 4-probe positive pressure Gilson GX-274 ASPEC $^{\mathrm{TM}}$ system (Gilson Inc., Middleton, WI, USA) with Discovery C18 $100 \mathrm{mg} / 1 \mathrm{~mL}$ solid phase extraction tubes (Supelco, St. Louis, MO, USA), using the following protocol: $3 \mathrm{~mL}$ of methanol was added for conditioning followed by $2 \mathrm{~mL}$ of $0.1 \%$ TFA in $\mathrm{H}_{2} \mathrm{O}$. The samples were then loaded onto each column followed by $4 \mathrm{~mL}$ of 95:5: $\mathrm{H}_{2} \mathrm{O}: \mathrm{ACN}, 0.1 \%$ TFA. Samples were eluted with $1 \mathrm{~mL}$ 80:20 ACN: $\mathrm{H}_{2} \mathrm{O}, 0.1 \%$ TFA. The samples were concentrated down to $\sim 100 \mu \mathrm{L}$ using a Speed Vac and a final $\mathrm{BCA}$ was performed to determine the peptide concentration, and then stored at $-80{ }^{\circ} \mathrm{C}$ until usage. Peptides digests were diluted to $0.1 \mu \mathrm{g} / \mathrm{uL}$ with nanopure water for LC-MS/MS analysis. $5 \mu \mathrm{L}$ of samples were loaded onto in-house packed reversed-phase capillary columns $(70-\mathrm{cm} \times 75 \mu \mathrm{m}$ i.d.) with $3-\mu \mathrm{m}$ Jupiter $\mathrm{C} 18$ particles. The separation was carried out using a nanoAcquity HPLC system (Waters Corporation, Milford, MA, USA) at room temperature. The mobile phase A was $0.1 \%$ formic acid in water while the mobile phase B was $0.1 \%$ formic acid in acetonitrile. The elution was carried out at $300 \mathrm{~nL} / \mathrm{min}$ with the following gradient: $0-2$ min $1 \%$ B; $2-20$ min $8 \%$ B; $20-75$ min $12 \%$ B; 75-97 $\min 30 \%$ B; $97-100 \min 45 \%$ B; $100-105 \min$ $95 \% \mathrm{~B} ; 110-140 \mathrm{~min} 1 \% \mathrm{~B}$. The eluting peptides were directly analyzed using a $Q$ Exactive Plus mass spectrometer (Thermo Fisher Scientific) in data-dependent acquisition mode. Mass spectrometer settings were as following: full MS (AGC, $3 \times 10^{6}$; resolution, 35,000; $\mathrm{m} / \mathrm{z}$ range, $400-2,000$; maximum ion time, $20 \mathrm{~ms}$ ); MS/ MS (AGC, $1 \times 10^{5}$; resolution, 17,500; $\mathrm{m} / \mathrm{z}$ range 200 2000; maximum ion time, $100 \mathrm{~ms}$; TopN, 12; isolation width, $2 \mathrm{Da}$; dynamic exclusion, $30.0 \mathrm{~s}$; collision energy, 
NCE 30). All mass spectrometry data were searched using MS-GF + and MASIC software. MS-GF + software was used to identify peptides by scoring MS/ MS spectra against peptides derived from the whole protein sequence database. MASIC software was used to generate the selected ion chromatographs (SICs) of all the precursors in MSMS datasets and calculate their peak areas as abundance. MASIC Results Merger (https://omics.pnl.gov/software/masic-results-merger) was used to append the relevant MASIC stats for each peptide-hit result in MS-GF+. The MS-GF + data were then filtered based on $1 \%$ false discovery rate (FDR) and less than $5 \mathrm{ppm}$ mass accuracy to generate a list of qualified peptide-hit results. The abundance of peptides was determined as the highest peak area identified for the peptide within a sample. Sample level quality was ensured by a Mahalanobis distance (rMd), which evaluates multiple statistical parameters associated with each peptide profile for each sample, as well as sample-level Pearson correlation and overall peptide intensity. Peptides were also filtered to remove those with inadequate data for statistics, where a peptide was required to have at least 2 observations in 2 of the groups in Table 1, or 3 observations within a single group. Protein quantification was performed using a standard reference-based approach. For time comparison within strains, we utilized two-sample t-tests with a Bonferroni correction. For strain comparison at the same time point, a oneway ANOVA with a Dunnett test was performed.

\section{Targeted mevalonate pathway metabolite analysis}

Strains $R$. toruloides WT, GB, GB2, and 312 were inoculated from LB precultures into SD media containing $20 \mathrm{~g} / \mathrm{L}$ glucose and a $20 \%$ (v/v) dodecane overlay. At $40 \mathrm{~h}$, ODs were measured and a $6 \mathrm{OD}_{600}$ cell pellet was collected from each culture by centrifugation in a microfuge at $5000 \mathrm{rpm}$ for $1 \mathrm{~min}$ at room temperature and then cells were quenched by adding $300 \mu \mathrm{L}$ of cold methanol. Samples were vortexed for $10 \mathrm{~s}$ and then $300 \mu \mathrm{L}$ chloroform was added, followed by another $10 \mathrm{~s}$ of vortexing. $150 \mu \mathrm{L}$ water was added, followed by another mix by vortexing for $10 \mathrm{~s}$. All solvents were liquid chromatography grade purity. Samples were centrifuged for $10 \mathrm{~min}$ at 14,000 rpm at $4{ }^{\circ} \mathrm{C}$ and the top aqueous layer was removed for metabolomics analysis. Water was added to bring the methanol/water ratio to $1: 1$ and samples were spun through Amicon 3000 molecular weight cut-off filters (Millipore Sigma, Burlington, MA, USA) and then lyophilized overnight and resuspended in $50 \mu \mathrm{L}$ of a sample solvent composed of $60 \%$ acetonitrile, $30 \%$ water, and $10 \%$ methanol. Samples were analyzed alongside mevalonate pathway standards using a hydrophilic interaction liquid chromatography time-of-flight mass spectrometry (HILIC-TOFMS) method described previously [26].

\section{Supplementary Information}

The online version contains supplementary material available at https://doi. org/10.1186/s13068-021-01950-w.

Additional file 1: Supplemental Figures and Tables.

\section{Acknowledgements}

We would like to thank the National Renewable Energy Lab for providing the DMR-EH corn stover hydrolysate.

\section{Authors' contributions}

$J K, G M G, J Y, J K, X Z$, JMS and JMG conceived and designed the study. JK, GMG, $J Y, J K, X Z, M B T, J P, E S, Y G, N M M, E E K B, V B$, and AF performed the experiments, collected and analyzed the data. JK, GMG, JK, and MBT wrote the manuscript, and all authors read, revised and approved the manuscript. JMG supervised the study. All authors read and approved the final manuscript.

\section{Funding}

This research was part of the Agile BioFoundry (https://agilebiofoundry. org) supported by the U. S. Department of Energy, Energy Efficiency and Renewable Energy, Bioenergy Technologies Office, through contract DE-AC02-05CH11231 between Lawrence Berkeley National Laboratory and the U. S. Department of Energy. The work conducted by the U.S. Department of Energy Joint Genome Institute, a DOE Office of Science User Facility, is supported by the Office of Science of the U.S. Department of Energy under Contract No. DE-AC02-05CH1 1231. A portion of this research was performed at Pacific Northwest National Laboratory (PNNL) using EMSL (grid.436923.9), a DOE Office of Science User Facility sponsored by the Office of Biological and Environmental Research. PNNL is a multiprogram national laboratory operated by Battelle for the Department of Energy (DOE) under Contract DE-AC05-76RL01830. Synthesis and assembly of DNA constructs by GenScript was supported in part by an Innovation Grant sponsored by GenScript Biotech Corporation. The views and opinions of the authors expressed herein do not necessarily state or reflect those of the United States Government or any agency thereof. Neither the United States Government nor any agency thereof, nor any of their employees, makes any warranty, expressed or implied, or assumes any legal liability or responsibility for the accuracy, completeness, or usefulness of any information, apparatus, product, or process disclosed, or represents that its use would not infringe privately owned rights. The Department of Energy will provide public access to these results of federally sponsored research in accordance with the DOE Public Access Plan (http:// energy.gov/downloads/doe-public-access-plan). Sandia National Laboratories is a multi-mission laboratory managed and operated by National Technology and Engineering Solutions of Sandia, LLC, a wholly owned subsidiary of Honeywell International, Inc., for the U.S. Department of Energy's National Nuclear Security Administration under contract DE-NA0003525.

\section{Availability of data and materials}

All data generated or analyzed during this study are included in this published article and its supplementary information files.

\section{Declarations}

Ethics approval and consent to participate Not applicable.

Consent for publication

Not applicable.

Competing interests

The authors declare no competing financial interests. 


\begin{abstract}
Author details
'Department of Energy, Agile BioFoundry, Emeryville, CA 94608, USA. ${ }^{2}$ Department of Biomass Science and Conversion Technology, Sandia National Laboratories, Livermore, CA 94550, USA. ${ }^{3}$ Joint BioEnergy Institute, Lawrence Berkeley National Laboratory, Emeryville, CA 94608, USA. ${ }^{4}$ Chemical and Biological Processing Group, Pacific Northwest National Laboratory, Richland, WA 99354, USA. ${ }^{5}$ Advanced Biofuels and Bioproducts Process Development Unit, Lawrence Berkeley National Laboratory, Emeryville, CA 94608, USA. ${ }^{6}$ Biological Systems and Engineering Division, Lawrence Berkeley National Laboratory, Berkeley, CA 94720, USA. ${ }^{7}$ Environmental Genomics and Systems Biology Division, Lawrence Berkeley National Laboratory, Berkeley, CA 94720, USA. ${ }^{8}$ QB3-Berkeley, University of California, Berkeley, CA 94704, USA. ${ }^{9}$ The Environmental Molecular Sciences Laboratory, Richland, WA 99354, USA.

${ }^{10}$ Biological Sciences Division, Pacific Northwest National Laboratory, Richland, WA 99354, USA.
\end{abstract}

\section{Received: 25 November 2020 Accepted: 7 April 2021}

Published online: 21 April 2021

\section{References}

1. Bouvier F, Rahier A, Camara B. Biogenesis, molecular regulation and function of plant isoprenoids. Prog Lipid Res. 2005:44:357-429.

2. Zhuang $X$, et al. Monoterpene production by the carotenogenic yeast Rhodosporidium toruloides. Microb Cell Fact. 2019;18:54.

3. Peralta-Yahya PP, et al. Identification and microbial production of a terpene-based advanced biofuel. Nat Commun. 2011;2:483.

4. Meadows AL, et al. Rewriting yeast central carbon metabolism for industrial isoprenoid production. Nature. 2016;537:694-7.

5. Geiselman GM, et al. Production of ent-kaurene from lignocellulosic hydrolysate in Rhodosporidium toruloides. Microb Cell Fact. 2020;19:24.

6. Yaegashi J, et al. Rhodosporidium toruloides: a new platform organism for conversion of lignocellulose into terpene biofuels and bioproducts. Biotechnol Biofuels. 2017;10:241.

7. Davari M, Ezazi R. Chemical composition and antifungal activity of the essential oil of Zhumeria majdae, Heracleum persicum and Eucalyptus sp. against some important phytopathogenic fungi. J Mycol Med. 2017:27:463-8.

8. Tomsheck AR, et al. Hypoxylon sp., an endophyte of Persea indica, producing 1,8-cineole and other bioactive volatiles with fuel potential. Microb Ecol. 2010;60:903-14.

9. Strobel GA. Bioprospecting-fuels from fungi. Biotechnol Lett. 2015:37:973-82.

10. Alonso-Gutierrez J, et al. Metabolic engineering of Escherichia coli for limonene and perillyl alcohol production. Metab Eng. 2013;19:33-41.

11. Mendez-Perez D, et al. Production of jet fuel precursor monoterpenoids from engineered Escherichia coli. Biotechnol Bioeng. 2017;114:1703-12.

12. Zebec $Z$, et al. Towards synthesis of monoterpenes and derivatives using synthetic biology. Curr Opin Chem Biol. 2016;34:37-43.

13. Chen $X$, et al. DMR (deacetylation and mechanical refining) processing of corn stover achieves high monomeric sugar concentrations $\left(230 \mathrm{~g} \mathrm{~L}^{-1}\right)$ during enzymatic hydrolysis and high ethanol concentrations (> 10\% $\mathrm{v} / \mathrm{v}$ ) during fermentation without hydrolysate purification or concentration. Energy Environ Sci. 2016;9:1237-45.

14. Zhang S, et al. Engineering Rhodosporidium toruloides for increased lipid production. Biotechnol Bioeng. 2016;113:1056-66.

15. Nora LC, et al. A toolset of constitutive promoters for metabolic engineering of Rhodosporidium toruloides. Microb Cell Fact. 2019;18:117.

16. Ham TS, et al. Design, implementation and practice of JBEI-ICE: an open source biological part registry platform and tools. Nucleic Acids Res. 2012:40:e141.

17. Trotta E. Selection on codon bias in yeast: a transcriptional hypothesis. Nucleic Acids Res. 2013;41:9382-95.

18. Tuller T, et al. An evolutionarily conserved mechanism for controlling the efficiency of protein translation. Cell. 2010;141:344-54.

19. Tuller T, Zur H. Multiple roles of the coding sequence $5^{\prime}$ end in gene expression regulation. Nucleic Acids Res. 2015;43:13-28.
20. Özaydın B, Burd H, Lee TS, Keasling JD. Carotenoid-based phenotypic screen of the yeast deletion collection reveals new genes with roles in isoprenoid production. Metab Eng. 2013;15:174-83.

21. Li H. Toward better understanding of artifacts in variant calling from highcoverage samples. Bioinformatics. 2014;30:2843-51.

22. Li H, et al. The sequence alignment/map format and SAMtools. Bioinformatics. 2009;25:2078-9.

23. Abyzov A, Urban AE, Snyder M, Gerstein M. CNVnator: an approach to discover, genotype, and characterize typical and atypical CNVs from family and population genome sequencing. Genome Res. 2011;21:974-84.

24. Kim D, Langmead B, Salzberg SL. HISAT: a fast spliced aligner with low memory requirements. Nat Methods. 2015;12:357-60.

25. Liao Y, Smyth GK, Shi W. featureCounts: an efficient general purpose program for assigning sequence reads to genomic features. Bioinformatics. 2014;30:923-30.

26. Baidoo EEK, Wang G, Joshua CJ, Benites VT, Keasling JD. Liquid chromatography and mass spectrometry analysis of isoprenoid intermediates in Escherichia coli. Methods Mol Biol. 2019;1859:209-24.

27. Rubat S, et al. Increasing the intracellular isoprenoid pool in Saccharomyces cerevisiae by structural fine-tuning of a bifunctional farnesyl diphosphate synthase. FEMS Yeast Res. 2017;17:54.

28. Stanley Fernandez SM, Kellogg BA, Poulter CD. Farnesyl diphosphate synthase. Altering the catalytic site to select for geranyl diphosphate activity. Biochemistry. 2000;39:15316-21.

29. Chen F, et al. Functional characterization of a geraniol synthase-encoding gene from Camptotheca acuminata and its application in production of geraniol in Escherichia coli. J Ind Microbiol Biotechnol. 2016;43:1281-92.

30. Zhang C, Li M, Zhao G-R, Lu W. Alpha-Terpineol production from an engineered Saccharomyces cerevisiae cell factory. Microb Cell Fact. 2019;18:160.

31. Paradise EM, Kirby J, Chan R, Keasling JD. Redirection of flux through the FPP branch-point in Saccharomyces cerevisiae by down-regulating squalene synthase. Biotechnol Bioeng. 2008;100:371-8.

32. Albermann S, Linnemannstöns P, Tudzynski B. Strategies for strain improvement in Fusarium fujikuroi: overexpression and localization of key enzymes of the isoprenoid pathway and their impact on gibberellin biosynthesis. Appl Microbiol Biotechnol. 2013;97:2979-95.

33. Xu J-W, Xu Y-N, Zhong J-J. Enhancement of ganoderic acid accumulation by overexpression of an $\mathrm{N}$-terminally truncated 3-hydroxy-3-methylglutaryl coenzyme A reductase gene in the basidiomycete Ganoderma lucidum. Appl Environ Microbiol. 2012;78:7968-76.

34. Ma SM, et al. Optimization of a heterologous mevalonate pathway through the use of variant HMG-CoA reductases. Metab Eng 2011;13:588-97.

35. Kazieva E, et al. Characterization of feedback-resistant mevalonate kinases from the methanogenic archaeons Methanosaeta concilii and Methanocella paludicola. Microbiology (Reading, Engl). 2017;163:1283-91.

36. Garcia DE, Keasling JD. Kinetics of phosphomevalonate kinase from Saccharomyces cerevisiae. PLoS ONE. 2014;9:e87112.

37. Tsay YH, Robinson GW. Cloning and characterization of ERG8, an essential gene of Saccharomyces cerevisiae that encodes phosphomevalonate kinase. Mol Cell Biol. 1991;11:620-31.

38. Geiselman $\mathrm{GM}$, et al. Conversion of poplar biomass into high-energy density tricyclic sesquiterpene jet fuel blendstocks. Microb Cell Fact. 2020;19:208.

39. Zada B, et al. Metabolic engineering of Escherichia coli for production of mixed isoprenoid alcohols and their derivatives. Biotechnol Biofuels. 2018;11:210

40. Wang CW, Oh MK, Liao JC. Engineered isoprenoid pathway enhances astaxanthin production in Escherichia coli. Biotechnol Bioeng. 1999;62:235-41.

\section{Publisher's Note}

Springer Nature remains neutral with regard to jurisdictional claims in published maps and institutional affiliations. 